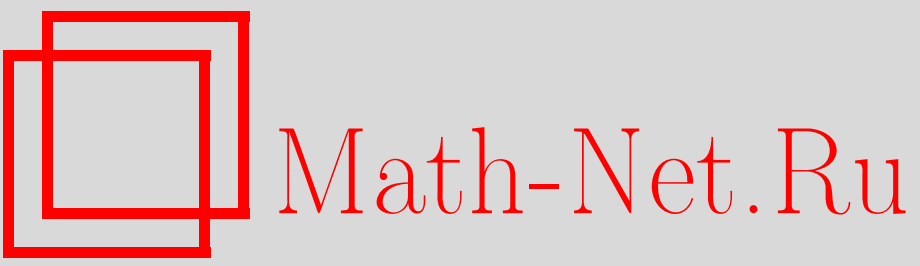

Ю. Г. Рудой, Обобщенная информационная энтропия и неканоническое распределение в равновесной статистической механике, ТМФ, 2003, том 135, номер 1, 3-54

DOI: https://doi.org/10.4213/tmf178

Использование Общероссийского математического портала Math-Net.Ru подразумевает, что вы прочитали и согласны с пользовательским соглашением

http: //www . mathnet.ru/rus/agreement

Параметры загрузки :

IP : 52.90 .164 .192

26 апреля 2023 г., 10:11:59

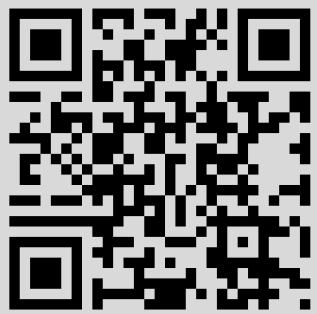


ТЕОРЕТИЧЕСКАЯ

И МАТЕМАТИЧЕСКАЯ

ФИЗИКА

Том 135, № 1

апрель, 2003

(C) 2003 г.

Ю. Г. Рудой*

\title{
ОБОБШЕННАЯ ИНФОРМАЦИОННАЯ ЭНТРОПИЯ \\ И НЕКАНОНИЧЕСКОЕ РАСПРЕДЕЛЕНИЕ В РАВНОВЕСНОЙ СТАТИСТИЧЕСКОЙ МЕХАНИКЕ
}

\begin{abstract}
На основе принципа Джейнса максимума информационной энтропии найдено обобщенное вероятностное распределение и построена обобщенная равновесная статистическая механика (РСM) для широкого класса объектов, к которым не применима обычная (каноническая) РСМ. Последовательно рассмотрен случай не дискретной, а непрерывной случайной переменной, характеризующей состояние объекта. Найденное распределение при больших значениях аргумента обладает не экспоненциальной, а степенной асимптотикой, которая соответствует эмпирически установленным закономерностям для подобных объектов. В качестве исходного энтропийного функционала использован $\varepsilon$-деформированный функционал Больцмана-Гиббса-Шеннона, удовлетворяющий требованиям энтропийной аксиоматики и при $\varepsilon=0$ приводящий к канонической РСМ; рассмотрены также нелинейные преобразования этого функционала. Показано, что в зависимости от способа определения средних значений динамических величин, характеризующих объект, при $\varepsilon \neq 0$ возможны различные варианты обобщенной РСМ (Цаллиса, Реньи, Харди-Литлвуда-Пойа) и дан их сравнительный анализ. Найдены условия выполнения термодинамических соотношений Гиббса-Гельмгольца и преобразования Лежандра для обобщенной энтропии и функции Масье-Планка. Подробно рассмотрены варианты РСМ по Цаллису и Реньи для случая одномерного вероятностного объекта с одной динамической величиной - обобщенной положительной "энергией", монотонно растущей по степенному закону. Получены ограничения на показатель Реньи, при которых равновесное распределение относится к определенному классу устойчивых распределений - Гаусса или Леви-Хинчина.
\end{abstract}

Ключевые слова: энтропия Шеннона, энтропия Реньи, энтропия Цаллиса, распределение Леви-Хинчина, принцип максимума энтропии Джейнса, равновесная статистическая механика.

\section{СОДЕРЖАНИЕ}

1. Введение . . . . . . . . . . . . . . . . . . . . . . . . . . . . . . . . .

2. Обобщенные средние значения и корреляционные функции . . . . . . . . . . 8

3. Принцип условного максимума информационной энтропии . . . . . . . . 10

Статья написана по заказу Редколлегии

\footnotetext{
* Российский университет дружбы народов, Москва, Россия. E-mail: ogol@mx.pfu.edu.ru
} 
4. Энтропия Больцмана-Гиббса-Шеннона и каноническая РСМ . . . . . . . . . 13

5. Обобшенное равновесное распределение . . . . . . . . . . . . . . . . . 15

6. Энтропийная аксиоматика для непрерывных распределений . . . . . . . . . . 19

7. Информационная функция и обобшенная энтропия Цаллиса . . . . . . . . . . 22

8. Обобшенная энтропия Реньи и Харди-Литлвуда-Пойа . . . . . . . . . . . . . . 28

9. Вариационная процедура для обобшенной энтропии и нелинейных средних зна-

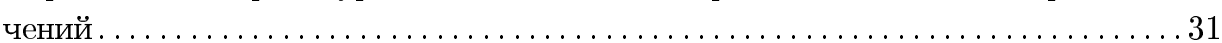

10. Обобшенные соотношения Гиббса-Гельмгольца . . . . . . . . . . . . . . . 33

11. Обобщенная РСМ для одномерного идеального газа. Общие соотношения . . . 35

12. Обобщенная РСМ для одномерного идеального газа. Канонический вариант и варианты Цаллиса и Реньи. . . . . . . . . . . . . . . . . . . . . . . . . . . . 39

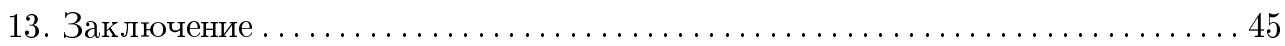

Приложение. Свойства обобщенного распределения . . . . . . . . . . . . . 47

\section{1. ВВЕДЕНИЕ}

Описание сложных открытых объектов различной природы - от естественнонаучных до социальных - является, как правило, не строго динамическим, но лишь вероятностным [1], [2] и дается функцией распределения $p(x, t)$, где $t$ - время, $x$ - дискретная или непрерывная величина, характеризуюшая положение объекта в его пространстве состояний $X$ или фазовом пространстве. Например, в физике в качестве величины $x$ могут рассматриваться координаты и импульсы частиц, в химии - концентрации реагентов, в биологии - числа в последовательности нуклеотидов в ДНК или аминокислот в белках, в экологии - численность особей в популяции и т.п.

Стохастическая динамика объекта, определяющая функцию распределения $p(x, t)$, описывается основным кинетическим уравнением или его упрошенной версией - уравнением Фоккера-Планка, справедливым в случае непрерывной величины $x$, если вероятности перехода между состояниями являются достаточно "гладкими"; однако полное решение этих уравнений для всех значений времени $t$ не всегда возможно.

Если интересоваться лишь состоянием статистического равновесия объектов, то достаточно ограничиться стационарной функцией распределения $p(x)$ (пределом $p(x, t)$ при $t \rightarrow \infty)$, для нахождения которой удобно использовать принцип максимума информационной энтропии $S$, впервые предложенный Джейнсом [3].

Согласно Больцману для физических объектов информационная энтропия с точностью до множителя совпадает с термодинамической энтропией Клаузиуса, так что принцип максимума следует из второго начала термодинамики. Для других вероятностных объектов этот принцип может рассматриваться как эвристическое обобщение. Для изолированного объекта имеет место абсолютный максимум $S$, соответствующий равновероятному распределению, а для открытого - условный; например, следует учесть условие сохранения среднего значения обобшенной “энергии” $E(x)$, которой объект обменивается с окружением (в физике эту роль играет термостат).

Таким образом, основная идея излагаемого подхода состоит в том, чтобы на основе феноменологического ( “термодинамического”) описания с помошью небольшого чис- 
ла эмпирически наблюдаемых для данного объекта параметров (например, объема $W$ пространства $X$, фиксированной средней “энергии” $E$ или сопряженного ему параметра “обратной температуры” $\beta$ ) построить $p(x)$. При этом может быть известно очень мало (или вообще ничего) о характере внутренних взаимодействий между составляющими объект элементами. Достаточно лишь того, чтобы эти взаимодействия обеспечивали устойчивость объекта и его “термализацию” за конечное время релаксации; кроме того, важно знать тип контакта объекта с внешним окружением.

Используя в качестве $S$ обычное выражение Больцмана-Гиббса-Шеннона, можно получить известное каноническое распределение, хорошо описываюшее большинство физических процессов (см., например, [4]). Однако во многих случаях это распределение обладает сушественным недостатком: при больших значениях $x$ оно имеет экспоненциальный характер и не описывает характерных для этих случаев степенных “хвостов" распределения. Наличие таких “хвостов" свидетельствует о важной роли больших флуктуаций и об отсутствии естественного масштаба для них (см. в этой связи, например, работы [5], [6]).

Примерами вероятностных объектов, характеризуюшихся степенными (неканоническими) распределениями, могут служить следующие объекты в естественных науках (подробнее см. [7], [8]): в ядерной физике - распределение адронов высоких энергий по поперечным импульсам [9], в астрофизике - распределение космических тел (от галактик до пыли) по массам, а также космических лучей по энергиям [9], [10], в геофизике распределение землетрясений по магнитудам (закон Гутенберга-Рихтера), в экологии распределение особей или видов по габаритам и массам (закон Уиллиса). Объекты подобного типа широко распространены также в социально-гуманитарных науках (см., например, [11]-[14]): в экономике - распределение социальных групп (от семьи до государства) по доходам (закон Парето), в демографии - распределение городов по численности населения (закон Юла), в наукометрии - распределение ученых по числу публикаций (закон Лотки), в лингвистике и теории информации - распределение слов связного текста (сообщения) по числу букв или знаков или частоте появления (закон Эсту-Ципфа-Мандельброта).

Объекты столь различной природы объединяет очень широкий интервал значений случайной переменной (как правило, 20-30 порядков величины, в астрофизике - до 40). В них также происходит своеобразная "конкуренция" за ограниченные (фиксированные в среднем) ресурсы, называемые обобщенной “энергией” (пища в экологии, деньги в экономике и т.п). При этом стационарные степенные распределения устанавливаются независимо от природы и конкретных деталей механизма случайных взаимодействий как внутри объекта, так и объекта с “термостатом".

Описанное "неканоническое" поведение может быть обусловлено такими факторами как негладкая (фрактальная) структура пространства состояний $X$ и (или) разрывный скачкообразный характер стохастической динамики в нем. Кроме того, как показали Монтролл и Шлезингер [15], подобное описание может быть актуально и для ряда физических объектов в условиях, когда существенным является учет пространственного дальнодействия (например, для самогравитирующих объектов) или временно́й памяти (например, в задачах аномальной диффузии). 
Оказывается, что класс неканонических распределений также может быть получен с помошью принципа максимума энтропии, если в качестве $S$ использовать отличную от энтропии Больцмана-Гиббса-Шеннона обобшенную энтропию, например введенную Реньи [16]. В дальнейшем Цаллис [17] предложил иной вид для $S$ и для случая дискретной переменной $x$ получил обобщенное распределение, которое в настоящее время широко используется [18], [19] $]^{1)}$. Обе энтропии содержат зависимость от параметра и в частном случае переходят в обычную энтропию Больцмана-Гиббса-Шеннона (соответственно обобщенное распределение переходит в каноническое).

Цель данной работы состоит в том, чтобы обобщить применение принципа Джейнса максимума информационной энтропии на случай непрерывной переменной $x$ (разделы 3-6), а затем на основе обобшенной энтропии Реньи и Цаллиса (разделы 7-9) построить два варианта равновесной статистической механики (РСМ) (разделы 10-12), т.е. найти соответствующие неканонические распределения и обобщенные средние значения. Кроме того, в разделах 11 и 12 для обоих вариантов проводится сравнительный анализ всех термодинамических следствий ${ }^{2)}$ для частного случая идеального газа со степенным законом зависимости обобщенной энергии от непрерывной переменной $x$.

Будем считать, что состояние вероятностного объекта (в дальнейшем для краткости - объекта) описывается непрерывной случайной переменной $x=\left(x^{1}, \ldots, x^{d}\right)$ в $d$-мерном $(d \geqslant 1)$ евклидовом пространстве $X$ состояний объекта. Здесь и далее переменная $x$ будет считаться безразмерной, т.е. выраженной в единицах какой-либо характерной для данного объекта величины размерности $x$. По аналогии с физическими объектами подобное описание естественно назвать микроописанием.

Стохастизация подобных объектов может быть обусловлена как наличием нелинейной внутренней динамики для величин $x(t)$, приводящей к динамическому хаосу, так и наличием неконтролируемого “теплового” взаимодействия объекта с внешним окружением ("термостатом").

Наиболее полное описание вероятностного объекта дается с помощью функции плотности распределения вероятностей $p(x)$ по состояниям $x$ (в дальнейшем для краткости называемой просто распределением). Эта функция, вообще говоря, зависит от времени $t$ (как явно, так и посредством зависимости $x(t)$ ); она может быть разрывной или даже сингулярной и в общем случае удовлетворяет лишь двум условиям - неотрицательности и нормируемости:

$$
p(x, t) \geqslant 0, \quad \int p(x, t) d x=1
$$

Интегрирование в (1) формально проводится по всему пространству $X$, но фактически ограничено областью доступных объекту состояний, для которых $p(x, t) \neq 0$,

$$
W=\int d x, \quad W \geqslant 1
$$

Объем $W$ этой области может быть и бесконечным.

\footnotetext{
1) Заметим, что возможны и другие способы выбора $S$ (см. [20]-[22]).

2) Ранее подобное сравнение не проводилось ввиду отсутствия термодинамики идеального газа по версии Реньи даже для случая дискретной переменной $x$.
} 
В начальный момент $t=0$ объект может находиться в состоянии с любой функцией распределения $p(x, 0)$. В частности, возможен предельньй случай вырожсенного, или дельтаобразного распределения

$$
p(x)=\delta\left(x-x_{0}\right)
$$

что формально соответствует возможности сколь угодно точного определения произвольного микросостояния $x_{0}$ объекта. Обсуждение этого вопроса для непрерывных распределений см. ниже в разделе 6.

Если объект изолирован от внешнего окружения, то по прошествии характерного для него времени "перемешивания" $\tau$ можно считать, что все его микросостояния (совместимые с точными законами сохранения) становятся практически равновероятными. Подобное предположение опирается на теорему Пуанкаре для консервативных динамических объектов и является обобщением гипотезы Больцмана о "молекулярном хаосе" для вероятностных объектов физической природы. Соответственно распределение $p(x, t \gg \tau)$ перестает зависеть от $x$ и $t$ и становится равномерным

$$
p(x)=\text { const }=W^{-1} \text {. }
$$

Любой объект в микросостоянии $x$ характеризуется набором небольшого числа $\partial u$ намических величин $A_{k}(x), k=1,2, \ldots$ Если объект составной, то величины $A_{k}(x)$ являются экстенсивными (пропорциональными числу составных частей объекта). Как правило, для изолированного (т.е. не взаимодействующего с термостатом) чисто динамического объекта величины $A_{k}(x)$ с течением времени сохраняются, т.е. являются точными интегралами движения $A_{k}(x)=$ const, что совместимо с микроописанием объекта.

В случае открытого (термодинамического) объекта в процессе его "теплового" взаимодействия с окружением происходит "обмен” динамическими величинами $A_{k}(x)$, нарушаюший их сохранение и делаюший их случайными. Поэтому открытый объект характеризуется уже не точными, а средними значениями $\bar{A}_{k}\{p(x, t)\}$ величин $A_{k}(x)$, которые являются функционалами распределения $p(x, t)$ (вообше говоря, зависяшими от времени) и определены ниже в разделе $2^{3}$. Соответственно для открытого вероятностного объекта становится возможным лишш макроописание.

После достаточно длительного (значительно превышающего все характерные для объекта времена релаксации) промежутка времени контакта между открытым объектом и окружением устанавливается “тепловое" (термодинамическое) равновесие. Подобное макроскопическое состояние объекта характеризуется равновесным распределением $p^{\mathrm{eq}}(x)$, а также набором нескольких сохраняющихся равновесных средних величин

$$
\bar{A}_{k}\left\{p^{\mathrm{eq}}(x)\right\}=A_{k}=\text { const } .
$$

\footnotetext{
3) Здесь и всюду в дальнейшем приняты следующие обозначения для аргументов: фигурные скобки - для аргумента $p(x)$ функционалов (энтропии $S$, средних $A_{k}$ и т.п.), квадратные скобки - для аргументов сложных функций и круглые - для простых.
} 
По предположению величины (5) являются экстенсивными термодинамическими параметрами, непосредственно измеряемыми макроскопическим наблюдателем. Конкретные значения величин (5) определяются условиями контакта на границе между вероятностным объектом и его окружением; важно, что в состоянии термодинамического равновесия эти значения постоянны ${ }^{4}$ ). Для дальнейшего (разделы 3 и 5 ) сушественно, что полностью эквивалентное описание этого состояния можно получить, фиксируя значения не самих экстенсивных величин $\left\{A_{i}\right\}$, а термодинамически сопряженных к ним интенсивных параметров $\left\{\alpha_{i}^{\mathrm{eq}}\right\}$ (см., например, [4]). При этом термодинамическая устойчивость состояния “теплового” равновесия обеспечивается знакоопределенностью термодинамических восприимчивостей (при всех $k$ )

$$
\chi_{k k} \equiv \partial A_{k} / \partial \alpha_{k}^{\mathrm{eq}}<0 .
$$

В частности, для физических объектов величины (6) связаны с теплоемкостью, сжимаемостью и т.п.

\section{2. ОБОБШЕННЫЕ СРЕДНИЕ ЗНАЧЕНИЯ И КОРРЕЛЯЦИОННЫЕ ФУНКЦИИ}

Средние значения $\bar{A}_{k}\{p(x, t)\}$ имеют смешанную статистико-динамическую природу и играют важную роль при построении РСМ. В обшем случае они представляют собой нелинейные функционалы от распределения $p(x)$, или взвешенные средние вида

$$
\tilde{\bar{A}}_{i}\{p(x)\}=\int A_{i}(x) \widetilde{\Pi}[p(x)] d x .
$$

Здесь $\widetilde{\Pi}(a)$ - всегда положительная “весовая” функция, или, как ее иногда называют (чтобы отличить от основного распределения $a \equiv p(x)$ ), сопровождающее вероятностное распределение. Здесь и далее для сокрашения записи сложные функции от распределения $p(x)$, где это возможно, будут записываться в виде простых функций от всегда положительного аргумента $a \equiv p(x)$. Предполагается, что в общем случае функция $\widetilde{\Pi}(a)$ зависит от $a$ нелинейно, например степенным образом (см. ниже разделы 7 и 9 ). Для любого варианта обобшенной РСМ функция П $(a)$ одинакова для всех $A_{k}(x)$, за исключением случая $k=0$ (см. сноску 4$)$, когда $\widetilde{\Pi}(a) \equiv a$.

Заметим, что определение (7) отличается от принятого в обычной теории вероятностей, однако широко используется при построении неканонической РСМ [16], [17], [22]. То же замечание справедливо относительно использования обобшенной (вообше говоря, неадлитивной) энтропии, выбор которой должен быть непременно согласован с выбором вида сопровождающего распределения (см. в этой связи, например, формулы (78) и (79) в разделе 7$)$.

Целесообразность определения (7) и нижеследующих формул может быть оправдана требованием разрешимости уравнения (23) для исходной функции распределения с условием (26) в рамках принципа условного максимума информационной энтропии (раздел 3 ) ; конкретную реализацию вариационной процедуры для различного выбора функционала энтропии см. в разделе 9 .

\footnotetext{
4) Формально удобно включить в число условий (5) при $k=0$ также условие нормировки (1), положив для этого $A_{0} \equiv 1$ при любой форме контакта и любом виде $p(x)$.
} 
Функция $\widetilde{\Pi}(a)$ и соответствуюшие ей средние $\tilde{\bar{A}}_{i}$ могут быть, вообше говоря, ненормированными, так что $\tilde{\overline{1}} \neq \tilde{1}$. Переход к нормированным средним значениям

$$
\bar{A}_{i}\{p(x)\}=\int A_{i}(x) \Pi[p(x)] d x
$$

возможен путем замены

$$
\widetilde{\Pi}(a) \rightarrow \Pi(a)=Q^{-1} \widetilde{\Pi}(a), \quad Q=\int \widetilde{\Pi}[p(x)] d x,
$$

где $Q>0, Q \neq 1$ - нормировочный множитель для сопровождающего распределения $\widetilde{\Pi}(a)$.

В случае, к которому относится, в частности, каноническая РСМ (см. ниже раздел 4), если это распределение оказывается линейным,

$$
\widetilde{\Pi}(a)=\Pi(a)=a, \quad Q=1,
$$

то оно совпадает с основным и в силу условия (1) всегда является нормированным. Для средних значений будем иметь

$$
\bar{A}_{i}\{p(x)\}=\int A_{i}(x) p(x) d x .
$$

В этом случае также сушествует лишь нормированный вариант.

Наряду со средними значениями (7) могут представлять интерес и корреляционные функции вида

$$
\sigma_{i j}\{p(x)\}=\int A_{i}(x) A_{j}(x) \widetilde{\Pi}[p(x)] d x
$$

или их нормированный вариант с П $(a)$ из выражения (9). Кроме того, могут представлять интерес и средние значения (моменты) более высоких степеней $\left(A_{k}(x)\right)^{n}(n \geqslant 2-$ целое число). В частности, при $n=2 \mathrm{c}$ их помощью определяется абсолютная $\partial u c n e p$ сия

$$
\sigma_{A_{k}}^{2} \equiv \overline{\left(\Delta A_{k}\right)^{2}}=\overline{A_{k}^{2}}-\bar{A}_{k}^{2},
$$

где

$$
\Delta A_{k} \equiv A_{k}(x)-\bar{A}_{k} \quad\left(\overline{\Delta A_{k}} \equiv 0\right)
$$

- флуктуация случайной величины $A_{k}(x)$ (в формулах $(13)-(16)$ и в ряде случаев в дальнейшем для простоты записи у средних значений $\bar{A}_{k}\{p(x)\}$ опушен функциональный аргумент $p(x))$.

Иногда удобнее пользоваться понятием относительной дисперсии

$$
\tilde{\sigma}_{A_{k}}^{2} \equiv \frac{\sigma_{A_{k}}^{2}}{\bar{A}_{k}^{2}}=\frac{\overline{A_{k}^{2}}}{\bar{A}_{k}^{2}}-1 .
$$

Согласно определениям (13) и (15) обе дисперсии при любом значении $k$ и любом распределении $p(x)$ должны быть положительны, так что

$$
\sigma_{A_{k}}^{2}>0 \quad \text { или } \quad \frac{\overline{A_{k}^{2}}}{\bar{A}_{k}^{2}}>1 .
$$


Это неравенство принято назьвать условием устойчивости объекта; его выполнение является критерием возможности построения того или иного варианта РСМ (заметим, что условие (16) эквивалентно условию (6) только в случае канонической РСМ - в обобщенных вариантах РСМ эти условия должны проверяться независимо, см. ниже разделы 10 и 11).

В обшем случае для всех $k \geqslant 1, n \geqslant 1(k, n$-целые числа) можно записать

$$
\overline{\left(A_{i}\right)^{n}}\{p(x)\}=\int\left(A_{i}(x)\right)^{n} \Pi_{n}[p(x)] d x
$$

Ясно, однако, что разумный смысл выражение (17) имеет лишш в том случае, когда функция $\Pi_{n}(a)$ не зависит от $n$, т.е. одинакова для всех моментов любого порядка $n$,

$$
\frac{\partial \Pi_{n}(a)}{\partial n} \equiv 0, \quad \Pi_{n}(a) \equiv \Pi(a)
$$

Для проведения в дальнейшем вариационной процедуры с участием функционалов средних значений, а также других функционалов (например, энтропии) представляют интерес функциональные производные от них по $p(x)$. Эти производные, которые являются уже не функционалами, а функциями $p(x)$, будут всюду обозначаться штрихами или верхним индексом $n$ (при $n \geqslant 3$ ), тогда как любые другие производные будут записываться в явном виде. Между обоими видами производных при любом $n \geqslant 1, k \geqslant 1$ имеется очевидная связь. Например, дифференцируя (8), находим

$$
\frac{\delta^{n} \bar{A}_{k}\{p(x)\}}{\delta p(x)^{n}} \equiv \bar{A}_{k}^{(n)}(a)=\left.A_{k}(x) \frac{\partial^{n} \Pi(a)}{\partial a^{n}}\right|_{a=p(x)}
$$

В линейном (в частности, каноническом) случае (11) выражение (19) упрощается и для всех $k$ (в том числе $k=0$ ) имеем

$$
\bar{A}_{k}^{\prime}=A_{k}(x), \quad \bar{A}_{k}^{(n)}=0 \quad(n \geqslant 2) .
$$

\section{3. ПРИНЦИП УСЛОВНОГО МАКСИМУМА ИНФОРМАЦИОННОЙ ЭНТРОПИИ}

“Термодинамически" равновесное распределение $p^{\mathrm{eq}}(x)$ для данного объекта с учетом дополнительных условий (5) может быть получено с помощью принципа условного максимума информационной энтропии. Этот принцип не является строго обоснованным (скорее, лишь эвристическим), но дает наиболее экономное описание вероятностного объекта. Джейнс [3] впервые применил этот принцип к открытым равновесным физическим объектам и весьма просто воспроизвел результаты статистической механики Гиббса; позднее Зубарев [4] успешно перенес указанный принцип на неравновесный случай.

Заметим, что в идейном плане Джейнс следовал Больцману, фактически впервые неявно применившему принцип максимума энтропии к изолированному физическому объекту типа классического идеального газа;в дальнейшем Планк, введя постоянную $k$, 
по существу, установил соответствие между термодинамической и информационной энтропиями.

После работ Шеннона [23] стало ясно, что формула Больцмана с точностью до множителя $k$ совпадает с формулой Хартли для максимального значения энтропии Шеннона при равномерном распределении (4), которое Больцман называл "молекулярным хаосом". Позднее Гиббс рассмотрел более обший случай произвольного значения "показателя фазы" (совпадающего с энтропией Шеннона) для любого динамического объекта.

Для применения принципа максимума информационной энтропии необходимо располагать соответствуюшим функционалом $S\{p(x)\}$; в отличие от средних величин (7), он всегда является нелинейным и не имеет никакого динамического "прообраза", поскольку величина $S\{p(x)\}$ характеризует только статистические свойства макросостояния объекта. На интуитивном уровне можно сказать, что величина $S\{p(x)\}$ характеризует степень "размытости" функции распределения $p(x)$, т.е. разброса объекта по его микросостояниям (или, иначе, степень "неопределенности" макросостояния объекта).

Вид функционала $S\{p(x)\}$ (особенно для непрерывной переменной $x$ ) не определяется вполне однозначно, но допускает значительный произвол, ограниченный лишь несколькими естественными требованиями, кратко называемыми энтропийной “аксиоматикой" (см., например, [20]-[26], а также ниже раздел 6).

Для физических объектов обычно используется "канонический” энтропийный функционал Больцмана-Гиббса-Шеннона

$$
S^{\mathrm{BGS}}\{p(x)\} \equiv S_{0}\{p(x)\}=-\int p(x) \ln p(x) d x .
$$

Нижний индекс 0 здесь и всюду далее обозначает принадлежность той или иной величины к каноническому варианту РСМ (единственное исключение - взаимно сопряженные величины $\alpha_{0}$ и $A_{0}$, описываюшие условие нормировки (1)). Однако в ряде случаев (например, для физических объектов, обладаюших фрактальной структурой пространства состояний $X$, а также вероятностных объектов другой природы) более адекватным может оказаться иной выбор (см., например, [16], [17], [21], [22]). В данной работе соответствуюший выбор производится в разделах 7 и 8 ; равновесное распределение на этой основе найдено в разделе 9 (его свойства подробно рассмотрены в разделе 5 и приложении), а различные варианты обобщенной РСМ построены в разделах 11 и 12.

Независимо от конкретного вида функционала $S\{p(x)\}$ реализация принципа условного максимума инфомационной энтропии сводится к вариационной процедуре нахождения безусловного максимума в пространстве распределений $\{p(x)\}$ функционала Лагранжа

$$
\mathcal{L}\{p(x)\}=S\{p(x)\}-\sum_{i \geqslant 0} \alpha_{i}\left[\bar{A}_{i}\{p(x)\}-A_{i}\right] .
$$

Наряду с функционалом $S\{p(x)\}$ в (22) входят дополнительные условия “термодинамического" равновесия (5), выражаюшиеся в виде линейной формы от функционалов $\bar{A}_{i}\{p(x)\}$ с неопределенными ("пробными") множителями Лагранжа $\left\{\alpha_{i}\right\}$ (соответственно $p(x)$ играет роль "пробной” функции распределения). 
Равновесное распределение $p^{\mathrm{eq}}(x)$ находится как решение уравнения экстремума для $\mathcal{L}\{p(x)\}$ в пространстве распределений $\{p(x)\}$,

$$
\mathcal{L}^{\prime}\left[p^{\mathrm{eq}}(x)\right]=0,
$$

где

$$
\mathcal{L}^{\prime}=S^{\prime}-\sum_{i \geqslant 0} \alpha_{i} \bar{A}_{i}^{\prime}=S^{\prime}-\sum_{i \geqslant 1} \alpha_{i} \bar{A}_{i}^{\prime}-\alpha_{0} .
$$

Уравнение (23) должно быть однозначно разрешимо в явном виде относительно $p^{\mathrm{eq}}(x)$, т.е функция (24) должна иметь обратную. Необходимым и достаточным условием этого является строгая монотонность функции (24) по аргументу $p(x)$, что, в свою очередь, означает знакоопределенность функциональной производной от (24),

$$
\mathcal{L}^{\prime \prime}=S^{\prime \prime}-\sum_{i \geqslant 0} \alpha_{i} \bar{A}_{i}^{\prime \prime}=S^{\prime \prime}-\sum_{i \geqslant 1} \alpha_{i} \bar{A}_{i}^{\prime \prime} .
$$

Поскольку нас интересует максимум функционала (22), то

$$
\mathcal{L}^{\prime \prime}[p(x)]<0
$$

при всех $p(x)$ (или, по крайней мере, при $p(x)=p^{\mathrm{eq}}(x)$ ), что означает выпуклость функционала (22). Заметим, что последнее условие устанавливает определенную связь между выбором функционалов $S$ и $A$, что будет использовано в дальнейшем (раздель $7-9$ ).

В данном разделе будем предполагать, что функционалы $\bar{A}_{i}\{p(x)\}$ являются линейным.ми и описываются формулой (11). Тогда изложенная выше схема несколько упрошается. Условие экстремума (23) принимает вид

$$
S^{\prime}\left[p^{\mathrm{eq}}(x)\right]-\alpha_{0}+b(x)=0,
$$

а условие выпуклости (26) переносится на энтропийный функционал,

$$
S^{\prime \prime}[p(x)]<0
$$

Входящая в (27) величина

$$
b(x) \equiv-\sum_{i \geqslant 1} \alpha_{i}^{\mathrm{eq}} A_{i}(x), \quad \frac{\partial b(x)}{\partial \alpha_{k}^{\mathrm{eq}}}=-A_{k}(x),
$$

является полилинейной формой как по динамическим переменным $A_{i}(x)$, так и по равновесным множителям Лагранжа $\alpha_{i}^{\mathrm{eq}}$ (ниже иногда вместо общего индекса "еq" будет указываться индекс, характеризующий данный вариант РСМ).

В дальнейшем важную роль играет среднее равновесное значение величины (29)

$$
-\bar{b}^{\mathrm{eq}}=\sum_{i \geqslant 1} \alpha_{i}^{\mathrm{eq}} \bar{A}_{i}\left\{p^{\mathrm{eq}}(x)\right\}=\sum_{i \geqslant 1} \alpha_{i}^{\mathrm{eq}} A_{i}, \quad \bar{b}^{\mathrm{eq}}=\bar{b}^{(0), \mathrm{T}, \mathrm{R}},
$$

причем верхний индекс у $b$ указывает, в рамках какого из вариантов РСМ происходит усреднение в (30). Равновесные значения $A_{i}$ определяются условиями $(5)$, а равновесные значения множителей Лагранжа $\alpha_{i}^{\text {eq }}$ неявным образом зависят от $A_{i}$, поскольку находятся подстановкой равновесного распределения $p^{\mathrm{eq}}(x)$ в дополнительные условия (5) и решения получаемой при этом системы уравнений $A_{i}=A_{i}\left\{\alpha_{k}^{\mathrm{eq}}\right\}$ (как правило, достаточно сложной). 


\section{4. ЭНТРОПИЯ БОЛЬЦМАНА-ГИББСА-ШЕННОНА И КАНОНИЧЕСКАЯ РСМ}

В качестве примера изложенной выше вариационной процедуры рассмотрим линейные средние значения (11) и канонический энтропийный функционал $(21)$, для которого

$$
S_{0}^{\prime}(a)=-(\ln a+1), \quad S_{0}^{\prime \prime}(a)=-\frac{1}{a}, \quad a \equiv p(x)
$$

Очевидно, что согласно второму из выражений (31) и первому из условий (1), условие выпуклости (28) выполняется для всех $p(x)$. Подставляя первое из выражений $(31)$ в уравнение экстремума (27), находим равновесное решение в виде нормированного экспоненциального распределения, или канонического распределения Гиббса,

$$
p_{0}^{\mathrm{eq}}(x)=Z_{0}^{-1}\left\{\alpha_{i}^{(0)}\right\} e^{b(x)}
$$

Заметим, что для частного случая, когда у простейшего физического объекта типа идеального газа имеется лишь одна динамическая переменная $A_{1}(x)$ - кинетическая или потенциальная энергия, а $\alpha_{1}=1 / k T$, где $T$ - абсолютная температура, подобное распределение фактически вводилось задолго до Гиббса сначала Максвеллом, а затем Больцманом. Величина $Z_{0}\left\{\alpha_{i}^{\mathrm{eq}}\right\}$, или статистический интеграл, вводится для большего удобства вместо множителя Лагранжа $\alpha_{0}$ (и, разумеется, связана с ним); в силу условия нормировки (второго из условий (1)) эта величина имеет вид

$$
Z_{0}\left\{\alpha_{i}^{(0)}\right\}=e^{1+\alpha_{0}}=\int e^{b(x)} d x
$$

Распределение (32) является сложной функцией от случайной переменной $x$, причем эта зависимость определяется всеми динамическими величинами $A_{i}(x)$, входяшими в $b(x)$. Таким образом, статистические свойства "термодинамически" равновесного вероятностного объекта оказываются тесно связанными с его динамическими свойствами. Кроме того, в (32) входит зависимость от "термодинамических" параметров $\alpha_{i}^{\mathrm{eq}}$, сопряженных к $A_{i}(x)$ и входящих как в $b^{\mathrm{eq}}(x)$, так и в $Z\left\{\alpha_{i}^{\mathrm{eq}}\right\}$.

Равновесную каноническую энтропию (для физических объектов с точностью до множителя $k$ совпадающую с термодинамической энтропией Клаузиуса) можно получить, подставляя распределение (32) в функционал (21),

$$
S_{0}=\ln Z_{0}\left\{\alpha_{i}^{(0)}\right\}-\bar{b}^{(0)}, \quad-\bar{b}^{(0)} \equiv \sum_{i \geqslant 1} \alpha_{i}^{(0)} A_{i}^{(0)} .
$$

Первое слагаемое в правой части (34) представляет собой каноническую функцию Масье-Планка

$$
\Phi_{0}\left\{\alpha_{i}^{(0)}\right\}=\ln Z_{0}\left\{\alpha_{i}^{(0)}\right\}=1+\alpha_{0},
$$

обладаюшую по определению производящим свойством

$$
\frac{\partial \Phi_{0}\left\{\alpha_{i}^{(0)}\right\}}{\partial \alpha_{k}^{(0)}}=-A_{k}^{(0)} .
$$


В этом нетрудно убедиться, дифференцируя выражение (33) по $\alpha_{k}$ с учетом (29). Тогда соотношение (34) можно записать в виде

$$
S_{0}\left\{A_{i}^{(0)}\right\}-\Phi_{0}\left\{\alpha_{i}^{(0)}\right\}=\sum_{i \geqslant 1} \alpha_{i}^{(0)} A_{i}^{(0)}
$$

и рассматривать как преобразование Лежандра от независимых переменных $\left\{\alpha_{i}^{(0)}\right\}$ к новым переменным $\left\{A_{i}^{(0)}\right\}$, для которых аналогичным $(36)$ производящим свойством обладает энтропия $S_{0}$,

$$
\frac{\partial S_{0}\left\{A_{i}^{(0)}\right\}}{\partial A_{k}^{(0)}}=\alpha_{k}^{(0)}
$$

Это свойство очень полезно, поскольку получить выражение для $S_{0}\left\{A_{i}^{(0)}\right\}$ значительно проще, чем непосредственно решать сложную систему неявных уравнений (5) относительно $\left\{\alpha_{i}^{(0)}\right\}$. Соотношения (36)-(38) принято называть соотношениями Гиббса-Гельмгольиа. Они составляют основу аппарата канонического варианта РСМ, и, как показано ниже в разделе 10, применимы также для обобшенных вариантов РСМ, основанных на принципе условного максимума информационной энтропии.

Наряду со средними значениями (36) и термодинамически сопряженными им параметрами (38) представляют интерес и обобщенные термодинамические восприимчивости

$$
\chi_{k m}^{(0)} \equiv \frac{\partial A_{k}^{(0)}}{\partial \alpha_{m}^{(0)}}=-\frac{\partial^{2} \Phi\left\{\alpha_{i}^{(0)}\right\}}{\partial \alpha_{m}^{(0)} \partial \alpha_{k}^{(0)}}
$$

и

$$
\psi_{m k}^{(0)} \equiv \frac{\partial \alpha_{m}^{(0)}}{\partial A_{k}^{(0)}}=\frac{\partial^{2} S_{0}\left\{A_{i}^{(0)}\right\}}{\partial A_{k}^{(0)} \partial A_{m}^{(0)}},
$$

причем матришы $\hat{\chi}^{(0)}$ и $\hat{\psi}^{(0)}$, очевидно, взаимно обратны. Аналогичные величины могут быть введены и в обобшенных вариантах РСМ. Однако, как будет показано ниже (разделы 10 и 11), связь $\chi$ с $\Phi$ имеет место всегда, тогда как связь $\psi$ с $S$ - только для канонического случая.

Специфика канонического случая (в которой можно убедиться непосредственно, используя соотношения (33)-(35), см. также [4]) состоит в том, что восприимчивости (39) совпадают с точностью до знака с корреляционными функциями (12) динамических величин,

$$
-\chi_{k m}^{(0)} \equiv{\overline{A_{k} A_{m}}}^{(0)}-\bar{A}_{k}^{(0)} \bar{A}_{m}^{(0)},
$$

что при $m=k$ дает абсолютные дисперсии (13) этих величин

$$
-\chi_{k k}^{(0)}=\sigma_{A_{k}^{(0)}}^{2}>0
$$

Следовательно, в каноническом случае условие термодинамической устойчивости (6) вполне эквивалентно условию (16). В обобщенном варианте РСМ это, вообще говоря, 
не так, поскольку равенства (41) и (42) не имеют места (подробнее см. разделы 11 и 12$)^{5}$. В этом случае восприимчивости (39) и дисперсии (13) необходимо находить независимо друг от друга, а условия устойчивости (6) и (16) проверять каждое по отдельности.

\section{5. ОБОБЩЕННОЕ РАВНОВЕСНОЕ РАСПРЕДЕЛЕНИЕ}

Рассмотрим уравнение экстремума (27) в более общем случае, когда энтропийный функционал $S\{p(x)\}$ не совпадает с каноническим $S_{0}\{p(x)\}$, но средние значения остаются линейными по $p(x)$. В этом случае решение для равновесной функции распределения может быть записано в виде, полностью аналогичном каноническому (32),

$$
p^{\mathrm{eq}}(x)=Z^{-1}\left\{\alpha_{i}^{\mathrm{eq}}\right\} \tilde{p}[b(x)],
$$

где

$$
Z\left\{\alpha_{i}^{\mathrm{eq}}\right\}=\int \tilde{p}[b(x)] d x .
$$

Единственное (но, как будет видно, весьма существенное) отличие распределения (43) от канонического (32) состоит в том, что функция $p[b(x)] \equiv p(b)$ не является экспонентой. Ее вид полностью определяется видом функционала $S\{p(x)\}$ (точнее, его производной $S^{\prime}[p(x)]$, входяшей в уравнение $\left.(27)\right)$. Важно, что функция $p(b)$ всегда обладает свойством

$$
\tilde{p}(0)=1 \text {. }
$$

Это обусловлено тем, что согласно определению (29) значение $b=0$ означает отсутствие каких-либо дополнительных условий вида (5), кроме условия нормировки (1), так что все $\alpha_{i}=0$ при $i \geqslant 1, \alpha_{0} \neq 0$. При этом распределение (43) естественным образом переходит в равномерное распределение (4), поскольку согласно (44) при условии (45) $Z=W$ (с учетом определения (2)).

Дифференцируя выражение (44) для статистического интеграла $n$ раз по любому из параметров $\alpha_{k}$ и учитывая линейность $(29)$ функции $b(x)$ по $\alpha_{k}$, получаем при всех $n \geqslant 0$

$$
\frac{\partial^{n} Z\left\{\alpha_{i}\right\}}{\partial \alpha_{k}^{n}}=(-1)^{n} \int\left[A_{k}(x)\right]^{n} \frac{\partial^{n} \tilde{p}[b(x)]}{\partial[b(x)]^{n}} d x .
$$

Очевидно, что при $n=0$ выражение (46) совпадает с исходным (44), а при $n \geqslant 1$ совпадает (с точностью до знака) с определением средних значений (17), причем $n$-я производная функции $\tilde{p}(b)$ по $b$ играет роль “весовой” функции $\widetilde{\Pi}_{n}$. Таким образом, соотношение (46) позволяет рассматривать величину $Z\left\{\alpha_{i}\right\}$ как производяшую функцию для простых (не центральных и ненормированных) моментов величины $A_{k}(x)$ (с точностью до знака и нормировочного множителя).

Аналогичным образом, дифференцируя по различным $\alpha_{k}$ и $\alpha_{l}$, можно получить и смешанные моменты величин $A_{k}(x)$ и $A_{l}(x)$ (корреляционные функции) вида (12). В частности, при $n=1$ правая часть (46) пропорциональна среднему значению (11), которое,

\footnotetext{
5) Как будет видно ниже (раздел 11, замечание после формулы (161)), указанное несовпадение обусловлено свойствами найденного в разделе 5 обобщенного равновесного распределения; это несовпадение исчезает при $\varepsilon=0$.
} 
по принятому в этом разделе предположению, является линейным функционалом от $p(x)$ (и, следовательно, от $\tilde{p}(x))$ :

$$
\bar{A}_{i}\left\{p^{\mathrm{eq}}(x)\right\}=\int A_{i}(x) p^{\mathrm{eq}}(x) d x=\frac{1}{Z} \int A_{i}(x) \tilde{p}(x) d x .
$$

Это означает, что истинной производящей функцией для первого (нормированного) момента $A_{k}(x)$ является функция $-\ln Z\left\{\alpha_{i}^{\mathrm{eq}}\right\}$, что согласуется с соотношениями (35) и (36), поскольку

$$
-\frac{\partial \ln Z\left\{\alpha_{i}\right\}}{\partial \alpha_{k}}=-\frac{1}{Z} \frac{\partial Z\left\{\alpha_{i}\right\}}{\partial \alpha_{k}} \equiv A_{k}\left\{p^{\mathrm{eq}}(x)\right\}=A_{k}
$$

Для производных более высокого порядка $n>1$ функция $-\ln Z\left\{\alpha_{i}\right\}$ будет производящей уже только для нормированных центральных моментов

$$
\overline{\left(\Delta A_{i}(x)\right)^{n}} \equiv \overline{\left(A_{i}(x)-A_{i}\right)^{n}},
$$

причем это свойство утрачивается для нелинейных по $p(x)$ средних.

Сравнение правых частей выражений (46) и (48) с учетом линейности средних (47) приводит к простому дифференциальному уравнению для $\tilde{p}(b)$,

$$
\frac{\partial \tilde{p}(b)}{\partial b}=\tilde{p}(b)
$$

Решение этого уравнения с граничным условием (45) определяет в качестве единственно возможного в рассматриваемом случае экспоненциальное распределение

$$
\tilde{p}_{0}(b)=e^{b}
$$

очевидным образом совпадаюшее с каноническим (32). Для распределения (51) $n$-я производная не зависит от $n^{6)}$. Согласно формуле (46) и в соответствии с условием (18) это позволяет использовать величину

$$
\frac{\partial^{n} \tilde{p}_{0}(b)}{\partial b^{n}}=\tilde{p}_{0}(b)
$$

в качестве $\widetilde{\Pi}_{n}\left(p_{0}\right)$. В данном (линейном) случае сопровождающее распределение при любом $n$ "вырождается" в основное.

С другой стороны, согласно уравнению (27) вид равновесного решения $p(b)$ определяется только видом энтропийного функционала $S\{p(x)\}$, точнее, его производной $S^{\prime}\{p(x)\}$, для которой $p$ и $\tilde{p}$ являются (с точностью до множителя $Z$ ) обратными функциями. Как показано выше в разделе 4 (формулы (31)-(33)), для получения канонического решения (51) необходимо использовать в качестве энтропийного функционал (21). Следует, однако, подчеркнуть, что этот вывод справедлив лиш при линейном по $p(x)$ определении средних значений (11). При более обшем определении возможны другие

\footnotetext{
6) Заметим, что подобным свойством обладает лишь каноническое распределение (51), что приводит в общем случае, например, к нарушению равенств (41) и (42).
} 
типы функционала $S\{p(x)\}$ и другие виды равновесных решений (43), однако при этом следует, вообше говоря, использовать общее уравнение экстремума (23).

Как будет показано ниже в разделах $7-9$, в принципе возможен такой взаимно согласованный выбор величин $S\{p(x)\}$ и $A_{k}\{p(x)\}$ (нелинейным образом зависящих от $p(x))$, при котором вариационная процедура приводит к решению той же структуры, что и $(43)$, но с $\tilde{p}(b) \neq \tilde{p}_{0}(b)$. При этом остаются в силе выражения (44)-(46), а величина $\partial \tilde{p}(b) / \partial b \neq \tilde{p}(b)$ играет роль сопровождаюшего распределения П $(a)$ для моментов первого порядка. Последнее, правда, верно лишь в том случае, если первая производная функции $\tilde{p}(b)$ каким-либо (пусть и нелинейньм) образом выражается через саму функцию $\tilde{p}(b)$.

Простейший после линейного (50) выбор при $n=1$ в (46) для обшего определения нелинейных ненормированных средних (7) состоит, очевидно, в следуюшей замене $p(b)$ в правой части $(50)$ :

$$
\frac{\partial \tilde{p}(b)}{\partial b}=[\tilde{p}(b)]^{q}, \quad q \neq 1 .
$$

Вешественное число $q$ (вообше говоря, произвольное) принято называть показателем Реньи (см. об этом ниже разделы $7,8,12$ ). В данной работе, однако, вместо $q$ всюду будет использоваться параметр $\varepsilon$-деформации канонического энтропийного функционала (21) (см. раздел 3), связанный с $q$ простым соотношением

$$
\varepsilon=q-1
$$

Решение уравнения (53) с граничным условием (45) имеет вид

$$
\tilde{p}_{q}(b)=[1+(1-q) b]^{1 /(1-q)}
$$

или

$$
\tilde{p}_{\varepsilon}(b)=[1-\varepsilon b]^{-1 / \varepsilon} .
$$

Видно, что обобшенное ненормированное распределение (55) при $q \rightarrow 1$ (или (56) при $\varepsilon \rightarrow 0$ ) непрерывным образом переходит в каноническое (51). То же, очевидно, имеет место и для производной функции (56) по $b$, поскольку

$$
\frac{\partial \tilde{p}_{\varepsilon}(b)}{\partial b}=\left[\tilde{p}_{\varepsilon}(b)\right]^{\varepsilon+1}, \quad \frac{\partial \tilde{p}_{0}(b)}{\partial b}=\tilde{p}_{0}(b) .
$$

Отметим также полезное свойство 7 )

$$
\left[\tilde{p}_{\varepsilon}(b)\right]^{-1}=\tilde{p}_{-\varepsilon}(-b), \quad\left[\tilde{p}_{0}(b)\right]^{-1}=\tilde{p}_{0}(-b) .
$$

Согласно исходному определению (53) $n$-я производная от $\tilde{p}_{\varepsilon}(b)$ по $b$ зависит от $n$ (см. формулу (П.8) приложения) и, следовательно, в качестве $\widetilde{\Pi}_{n}\left(\tilde{p}_{\varepsilon}\right)$ использоваться не может. Таким образом, величина $Z$ (или $-\ln Z$ ) при $n>1$ теряет свое производящее

\footnotetext{
7) Ряд других свойств обобщенного распределения (56), отсутствующих у канонического распределения (51), рассмотрены в приложении.
} 
свойство, которым она обладала при $\varepsilon=0$. В качестве сопровождающего распределения может быть использована лишь величина

$$
\Pi_{1}\left(p_{\varepsilon}\right)=\left(\tilde{p}_{\varepsilon}\right)^{\varepsilon+1}=Z_{\varepsilon}^{\varepsilon+1} p_{\varepsilon}
$$

Условием вешественности и положительности решения (56) при любых значениях $b$ и $\varepsilon$ является выполнение неравенства

$$
1-\varepsilon b>0
$$

которое становится тем более ограничительным для $\varepsilon$, чем больше $|b|$. Другие (как правило, более жесткие чем (60)) ограничения возникают из условий существования статистического интеграла (44) и устойчивости (16) (см. раздел 11). Действительно, при $|b| \rightarrow 0$ неравенство (60) справедливо практически при всех вешественных значениях $\varepsilon$ (в частности, при $|b|=0$ имеет место свойство (45)). Однако при $|b| \rightarrow \infty$ ситуация существенно изменяется, и при $b>0$ для $\varepsilon$ заведомо допустимой является неположительная полуось $\varepsilon \leqslant 0$, тогда как при $b<0$ - соответственно неотрицательная полуось $\varepsilon \geqslant 0$. Строго говоря, в обоих случаях имеет место “заход" параметра $\varepsilon$ на другую полуось, однако величина этого “захода" $|\Delta \varepsilon|=|b|^{-1}$ и не играет сколько-нибудь заметной роли при $|b| \rightarrow \infty$.

Случай

$$
b<0, \quad \varepsilon \geqslant 0
$$

представляется более интересным для приложений РСМ, поскольку при $\varepsilon \neq 0$ для него характерна нетривиальная асимптотика распределения (56) (см. формулу (П.24)), отсутствуюшая при $\varepsilon=0$. В дальнейшем мы ограничимся только случаем (61), который реализуется, когда все $\alpha_{i}$ и $A_{i}(x)$ в (29) положительны. В случае $b>0, \varepsilon \leqslant 0$ величины $\alpha_{i}$ и $A_{i}(x)$ могут иметь разные знаки, что иногда создает дополнительные трудности, связанные со сходимостью статистического интеграла (44). В простейшем случае это означает положительность температуры и наличие отталкивающего потенциала, тогда как для $b<0$ - как правило, наличие притягивающего потенциала или отрицательность температуры.

В заключение этого раздела заметим, что способ получения обобшенного распределения (56), основанный на дифференциальном уравнении (53) с граничным условием (45) (или эквивалентном ему функциональном уравнении (П.6)) не может рассматриваться как последовательный, и в рамках излагаемого подхода он может быть использован лишь в качестве наводяшего соображения. Дальнейшая задача состоит в том, чтобы построить такой энтропийный функционал, для которого распределение (56) было бы решением уравнения экстремума (23) с условием (26), причем указанный функционал удовлетворял бы требованиям энтропийной “аксиоматики” (раздел 6), а средние значения определялись с помощью соответствуюшего нелинейного функционала вида (7) или (8). Ниже в разделе 7 будет показано, что подобным функционалом является энтропия Цаллиса, получаемая из энтропии Больцмана-Гиббса-Шеннона (21) посредством операции " $\varepsilon$-деформирования", а соответствующие ненормированные средние значения (7) определяются с помошью сопровождаюшего распределения (59). 


\section{6. ЭНТРОПИЙНАЯ АКСИОМАТИКА ДЛЯ НЕПРЕРЫВНЫХ РАСПРЕДЕЛЕНИЙ}

Для того чтобы функционал $S\{p(x)\}$ мог играть роль информационной әнтропии для любых непрерывных вероятностных распределений, подчиняюшихся лишь условиям (1), он должен удовлетворять ряду естественных требований. Эти требования обеспечивают наличие у энтропийного функционала достаточно "хороших" свойств, позволяющих придать ему в дальнейшем физический смысл. Соответствующие требования и свойства в основном заимствованы у энтропии Шеннона $S\left\{p_{i}\right\}$ для дискретного случая

$$
S\left\{p_{i}\right\}=-\sum_{i=1}^{W} p_{i} \ln p_{i} .
$$

В аксиоматике Хинчина [24] (см. также [25]) энтропия Шеннона [23] определяется однозначно. Однако, как показано, например, в [22] (см. также [20]), при замене всего лишь одной (4-й) аксиомы можно получить более общую энтропию Реньи [16]. Мы не будем опираться на указанные математические результаты и сформулируем здесь аналогичные требования на менее строгом уровне.

В случае (62) вероятностный объект имеет конечное или счетное число состояний $W$, нумеруемых индексом $i$ и характеризуемых набором неотрицательных вероятностей $\left\{p_{i}\right\}$ (нормированных и в отличие от непрерывного случая ограниченных сверху),

$$
0 \leqslant p_{i} \leqslant 1, \quad \sum_{i=1}^{W} p_{i}=1 .
$$

Нетрудно видеть, что при вьполнении этих условий энтропия (62) всегда неотрицательна, а благодаря выпуклости функции - $p \ln p$ и неравенству Иенсена ограничена также и сверху (при условии конечности величины $W$ ), т.е.

$$
0 \leqslant S\left\{p_{i}\right\} \leqslant \ln W
$$

При этом нижний и верхний пределы для энтропии Шеннона достигаются соответственно для экстремальных распределений: вырожденного $p_{i}=\delta_{i i_{0}}\left(i_{0}-\right.$ любое состояние) и равномерного $p_{i}=$ const $=1 / W$ (эти распределения являются дискретными аналогами распределений (3) и (4)).

Прямое обобшение выражения (62) на непрерывный случай - так называемая абсолютная энтропия, или энтропийный функционал (21) с доопределением $0 \ln 0=0$ - не обладает некоторыми из перечисленных вьше свойств. Прежде всего, отсутствует неотрицательность (и вообще ограниченность как снизу, так и сверху) ${ }^{8}$. Кроме того, функционал (21) не инвариантен относительно невырожденного преобразования исходной случайной переменной $x$. Поэтому согласно [26] (см. также [27], [28]), выражение (21)

\footnotetext{
8)Утрата свойства ограниченности снизу энтропии как меры неопределенности в непрерывном случае вполне естественна, поскольку информация Шеннона $\Delta I=-\Delta S$ (в битах или натах), необходимая для сколь угодно точного задания точки континуума, может быть по определению сколь угодно велика (см. [27]).
} 
должно быть заменено соответствующей относительной (называемой также условной, дифференциальной или приведенной) энтропией

$$
S_{\text {отн }}^{\mathrm{BGS}}\{p(x)\}=-\int p(x) \ln \frac{p(x)}{\mu(x)} d x,
$$

где $\mu(x)$ - неотрицательная и достаточно гладкая функция, которая при вычислении функциональных производных величины (65) по $p(x)$ рассматривается как постоянная.

Простейшим и наиболее естественным выбором этой функции (см., например, [27]) может служить

$$
\mu(x)=(\Delta x)^{-1}, \quad \Delta x>0,
$$

где $\Delta x$ - предельно возможная для данного объекта в данных условиях точность "микроскопического" задания (или, что то же, измерения) величины $x$, здесь и далее предполагаемой безразмерной. Тем самым фактически производится “дискретизация" пространства $X$ или, что то же, крупноструктурное усреднение функции распределения в духе Эренфеста (подробнее см. [4]), что обеспечивает выполнение для энтропии $H$-теоремы Больцмана. Кроме того, за счет слагаемого $\ln \Delta x$ достигается необходимый сдвиг начала отсчета энтропии.

Наличие конечной строго положительной величины $\Delta x$ обусловлено чисто физическими причинами (не обязательно квантовой природы), которые в принципе ограничивают точность измерения любой непрерывной (в математическом смысле) величины $x$. Конкретный выбор значения $\Delta x$ диктуется характером распределения $p(x)$ безразмерной величины $x$. В частности, в случае достаточно “широкого" и гладкого распределения можно положить $\Delta x=1$, чем мы фактически и ограничимся в данной работе. С другой стороны, для “узкого” и сингулярного распределения в пределе малой дисперсии $\eta \rightarrow 0$ целесообразно положить $\Delta x \approx \eta^{1 / 2}$, что компенсирует возможные расходимости в правой части (21) и позволяет восстановить ограниченность снизу этого функционала. В этом нетрудно убедиться, если рассматривать вырожденное дельтаобразное распределение (3) как предел по соответствующему параметру $m \rightarrow \infty$ какого-либо подходящего одномодового распределения ${ }^{9)} p_{m}(x) \rightarrow \delta\left(x-x_{0}\right)$, для которого в этом пределе "мода" (точка максимума) $x_{0}^{(m)} \rightarrow x_{0}$, а дисперсия $\eta_{m} \rightarrow 0$. В том же предельном смысле здесь и ниже (разделы 6-8) следует понимать и любые функционалы от такого распределения.

Условие нормировки (1) выполняется при любом значении $m$ (формально и при $m=$ $\infty)$. Однако в контексте изложенных выше соображений реальный физический смысл имеют лишш распределения $p_{m}(x)$ со сколь угодно большим, но конечным значением $m$. Соответственно дисперсия $\eta_{m}$ и величина $\Delta x_{m} \approx\left(\eta_{m}\right)^{1 / 2}$ могут быть сколь угодно малыми, но отличными от нуля (например, для распределений, перечисленных в сноске 9 , $\left.\eta_{m} \sim m^{-2}, \Delta x_{m} \sim m^{-1}\right)$.

\footnotetext{
9) В одномерном случае в качестве таких распределений могут быть, например, использованы следующие: при всех действительных $x$ - "ступенчатое" распределение или нормальное распределение Гаусса, а при неотрицательных $x \geqslant 0$ - гамма-распределение или распределение Вейбулла-Гнеденко [28].
} 
В дальнейшем для проведения описанной в разделе 3 вариационной процедуры будет использован функционал информационной энтропии $S\{p(x)\}$ более общего вида, чем (65). С учетом сформулированных выше условий на выбор функции $\mu(x)$ вида (66) естественно ожидать выполнение для этого функционала следуюших требований.

1. Гладкость в пространстве распределений $\{p(x)\}$, а именно непрерывность и дважды дифференцируемость.

2. Экстремальность для предельных видов распределений ${ }^{10)}$ :

а) абсолютный минимум при вырожденном (сингулярном) распределении (3)

$$
S\left\{\delta\left(x-x_{0}\right)\right\}=S^{\min }\left(x_{0}\right), \quad \frac{\partial S^{\min }\left(x_{0}\right)}{\partial x_{0}}=0 ;
$$

б) абсолютный максимум при равномерном (равновероятном) распределении (4)

$$
S\left\{\frac{1}{W}\right\}=S^{\max }(W), \quad \frac{\partial S^{\max }(W)}{\partial W}>0 .
$$

3. Аддитивность для статистически независимых объектов:

$$
S\{p(x)\}=S\left\{p\left(x_{1}\right)\right\}+S\left\{p\left(x_{2}\right)\right\},
$$

если совместное распределение вероятностей мультипликативно, т.е.

$$
\begin{gathered}
p(x)=p\left(x_{1}\right) p\left(x_{2}\right), \quad x \in X, \\
x_{1} \in X_{1}, \quad x_{2} \in X_{2}, \quad X=X_{1} \cup X_{2}, \quad X_{1} \cap X_{2}=0 .
\end{gathered}
$$

Существенно, что для обобщенного распределения (56) (в отличие от канонического случая (51)) из статистической независимости (70) не следует, вообще говоря, $\partial u$ намическая независимость, под которой понимается адлитивность всех характеризуюших объект динамических величин (подробнее см. раздел 1 приложения),

$$
A_{k}(x)=A_{k}\left(x_{1}\right)+A_{k}\left(x_{2}\right) .
$$

Действительно, как видно из (56) и (29), обобщенное равновесное распределение связывает воедино статистические $(\alpha)$ и динамические $(A(x))$ свойства объектов. Произведение таких распределений дается формулой (П.6). Поскольку объекты предполагаются совершенно одинаковыми, то в одних и тех же условиях равновесные значения интенсивных термодинамических параметров для них одинаковы, т.е.

$$
\left(\alpha_{k}^{\mathrm{eq}}\right)^{(1)}=\left(\alpha_{k}^{\mathrm{eq}}\right)^{(2)}=\alpha_{k}^{\mathrm{eq}} .
$$

Тогда, как видно из выражений (П.2)-(П.7), свойство (71) следует из свойства (70) (и наоборот) лишш в каноническом случае $\varepsilon=0$.

Таким образом, неаддитивность динамических величин для статистически независимых объектов в случае обобшенного распределения может иметь место даже тогда, когда энтропия этих объектов является аддитивной.

\footnotetext{
10) Именно с этим свойством связано наименование энтропии как информационной: согласно определению Шеннона, информация $I=S_{\max }-S$ достигает максимума при $S=0$ в состоянии объекта (3) и минимума при $S=S_{\max }$ в состоянии объекта (4).
} 


\section{7. ИНФОРМАЦИОННАЯ ФУНКЦИЯ И ОБОБШЕННАЯ ЭНТРОПИЯ ЦАЛЛИСА}

Представим искомый функционал для абсолютной энтропии в виде (см., например, $[20],[28])$

$$
S\{p(x)\}=\int F[p(x)] d x
$$

где $F(a)$ - информационная функиия, определенная для всех неотрицательных значений $a \equiv p(x)$ (соответственно для относительной энтропии $a \equiv p(x) / \mu(x)^{11)}$ по аналогии с (65) с учетом (66)). Заметим, что представление (73) отнюдь не является необходимым свойством энтропийного функционала. В этом качестве могут, например, использоваться также какие-либо функции от левой части (73) при заданном виде $F$ (см. раздел 8). Представление (73) позволяет заменить функциональные производные $S\{p(x)\}$ по $p(x)$ обычными производными $F(a)$ по $a$,

$$
S^{(n)}[p(x)]=\left.\frac{\partial^{n} F(a)}{\partial a^{n}}\right|_{a=p(x)} .
$$

Выбор информационной функции $F(a)$ должен быть согласован с требованиями 1-3 раздела 6. В частности, для канонического функционала (21) эта функция имеет вид

$$
F_{0}(a)=-a \ln a, \quad a>0
$$

а ее производные даются выражениями (31).

Для того чтобы выйти за рамки канонического равновесного распределения (51) и получить, в частности, степенную асимптотику (П.24) вместо экспоненциальной (П.23), необходимо модифицировать логарифмический множитель в (75). Подобную модификацию, как и вообще выбор функции $F(a)$, можно осуществить различными способами (см., например, [20]-[22]). Наиболее естественно применить к “канонической” функции $F_{0}(a)$ операцию $\varepsilon$-деформирования, позволяющую получить вместо (75) однопараметрическое семейство информационных функций $F_{\varepsilon}(a)$. Тем самым будет продолжена реализация программы Фаддеева [29] по построению физических теорий “общего положения" (в этом смысле согласно предложенной в [29] терминологии каноническая РСМ является "вырожденной" теорией, соответствующей значению $\varepsilon=0$ ).

Будем рассматривать $\ln a$ как предельную функцию при любом $a>0$, используя для нее известное представление

$$
\ln a \equiv \Psi_{0}(a)=\lim _{\varepsilon \rightarrow 0} \Psi_{ \pm \varepsilon}(a)
$$

где $\varepsilon$ - любое вешественное число,

$$
\Psi_{ \pm \varepsilon}(a)= \pm \frac{1}{\varepsilon}\left(a^{ \pm \varepsilon}-1\right), \quad \Psi_{-\varepsilon}(a)=-\Psi_{\varepsilon}\left(a^{-1}\right) .
$$

\footnotetext{
11) Напомним, что выбор $\mu(x) \neq 1$ необходим только для сингулярных распределений $p_{m}(x) \rightarrow$ $\delta\left(x-x_{0}\right)$ (при $m \rightarrow \infty$ ). В этом случае он приводит к регуляризации энтропии и сдвигу (возможно, бесконечному) начала отсчета для нее (см. ниже формулы $(95),(127)$ и (128)).
} 
Заметим, что аналогичный прием используется, например, в методе реплик для вычисления логарифма статистической суммы спинового стекла [30]. Сушественно, что $\Psi_{0}$ можно выразить через $\Psi_{\varepsilon}$ и без перехода к пределу $\varepsilon \rightarrow 0$ при любом конечном $\varepsilon$ посредством преобразования Реньи (см. ниже (113)). Выбирая в (76) для определенности верхний знак, имеем

$$
F_{\varepsilon}(a)=-a \Psi_{\varepsilon}(a)=-\frac{1}{\varepsilon}\left[P_{\varepsilon}(a)-a\right],
$$

где

$$
P_{\varepsilon}(a) \equiv a^{\varepsilon+1}, \quad P_{-1}(a)=1, \quad P_{0}(a)=a .
$$

Функция (79) всегда положительна (в силу первого из условий (1)); тем же свойством обладает функционал

$$
Q_{\varepsilon}\{p(x)\}=\int P_{\varepsilon}[p(x)] d x, \quad Q_{\varepsilon}>0,
$$

функциональные производные которого имеют вид

$$
Q_{\varepsilon}^{(n)}[p(x)]=\left.\frac{\partial^{n} P_{\varepsilon}(a)}{\partial a^{n}}\right|_{a=p(x)} .
$$

Функция $P_{\varepsilon}[p(x)]$, играюшая роль сопровождаюшего вероятностного распределения, и функционал $Q_{\varepsilon}\{p(x)\}$, являюшийся его нормировкой, очень важны для дальнейшего изложения. Именно с их помошью при $p(x)=p^{\text {eq }}(x)$ выражаются обобшенные средние значения и обобщенная равновесная энтропия, соответственно.

Функционал (80) обладает следуюшими свойствами. В силу второго из условий (1) и определения (2) для любых распределений $p(x)$ справедливы равенства

$$
Q_{-1}\{p(x)\}=W, \quad Q_{0}\{p(x)\}=1 .
$$

Кроме того, для экстремальных распределений (3) и (4) функционал (80) при любом $\varepsilon$ и больших, но конечных $m$ и $W$ принимает следующие значения:

$$
Q_{\varepsilon}\left\{p_{m}(x)\right\} \sim m^{\varepsilon}, \quad Q_{\varepsilon}\left\{\frac{1}{W}\right\}=W^{-\varepsilon} .
$$

Заметим, что в соответствии с формулами (82) при $\varepsilon=0$ имеем $Q_{0}=1$ при любом значении $m$, тогда как при $\varepsilon>0$ величина $Q_{\varepsilon}$ расходится, когда $m \rightarrow \infty$.

Функция $F_{\varepsilon}(a)$ при любом $\varepsilon$ является знакопеременной,

$$
F_{\varepsilon} \gtrless 0 \quad(a \lessgtr 1), \quad F_{\varepsilon}(1)=F_{\varepsilon}(0)=0,
$$

а ее производные по $а$ имеют вид

$$
\frac{\partial F_{\varepsilon}(a)}{\partial a}=S^{\prime}(a)=-\frac{1}{\varepsilon}\left[\frac{\partial P_{\varepsilon}(a)}{\partial a}-1\right]
$$

или

$$
\frac{\partial F_{\varepsilon}(a)}{\partial a}=-\left[\Psi_{\varepsilon}(a)+a^{\varepsilon}\right]=-\left(\frac{\varepsilon+1}{\varepsilon}\right) a^{\varepsilon}+\frac{1}{\varepsilon},
$$


а также

$$
\frac{\partial^{2} F_{\varepsilon}(a)}{\partial a^{2}}=S^{\prime \prime}(a)=-\frac{1}{\varepsilon} \frac{\partial^{2} P_{\varepsilon}(a)}{\partial a^{2}}=-(\varepsilon+1) a^{\varepsilon-1} .
$$

Нетрудно видеть, что выражения (78), (85)-(87) при $\varepsilon=0$ переходят соответственно в выражения (75) и (31) для канонического случая.

Сопоставляя формулы (81), (85) и (87), находим связь между функциональными производными $S_{\varepsilon}\{p(x)\}$ и $Q_{\varepsilon}\{p(x)\}$ при всех $n \geqslant 2$,

$$
S_{\varepsilon}^{\prime}(a)=-\frac{1}{\varepsilon}\left[Q_{\varepsilon}^{\prime}(a)-1\right], \quad S_{\varepsilon}^{(n)}(a)=-\frac{1}{\varepsilon} Q_{\varepsilon}^{(n)}(a), \quad a \equiv p(x) .
$$

Предположим, что выполняется условие ограничения на параметр деформации $\varepsilon$ (или, что то же, на показатель Реньи $q=\varepsilon+1)$,

$$
\varepsilon+1>0 \quad \text { или } \quad q>0 \text {. }
$$

Тогда функция $F_{\varepsilon}(a)$, а следовательно, и функционал энтропии $S_{\varepsilon}\{p(x)\}$ обладает согласно (87) свойством выпуклости при всех $a$

$$
S_{\varepsilon}^{\prime \prime}(a)=-\frac{1}{\varepsilon} Q_{\varepsilon}^{\prime \prime}(a)<0
$$

Подставляя информационную функцию $F_{\varepsilon}[p(x)]$ в виде $(78)$ в общее выражение $(73)$ и учитывая при интегрировании определение (80) и второе из условий $(1)$, находим для обобщенного энтропийного функционала выражение

$$
S_{\varepsilon}^{\mathrm{T}}\{p(x)\}=-\frac{1}{\varepsilon}\left[Q_{\varepsilon}\{p(x)\}-1\right],
$$

которое было впервые предложено без какого-либо обоснования Цаллисом [17] в качестве основы для построения обобшенной РСМ. Обсуждение связанных с выражением (91) аспектов стало предметом весьма многочисленных работ (см., например, обзоры [18], [19]). В дальнейшем там, где это не вызовет недоразумений, верхний индекс Т будет для краткости опускаться.

Выражение (91) удобно для нахождения значений $S_{\varepsilon}$ при различных значениях $\varepsilon$ и разном виде $p(x)$. Однако при анализе обших свойств $S_{\varepsilon}$ предпочтительнее пользоваться общим представлением (73) с учетом (78). Так, например, дифференцируя (91) по $p(x)$, получаем выражение, отличающееся от правильного (85) на слагаемое $1 / \varepsilon$, что сушественно при переходе к пределу $\varepsilon=0$ (это слагаемое “теряется" при интегрировании в процессе перехода от (78) к (73) благодаря учету условия нормировки (1)).

Кроме того, предельный переход от энтропии Цаллиса к канонической энтропии Больцмана-Гиббса-Шеннона, элементарный в обшем выражении (73) с учетом определения (78), в выражении (91) требует раскрытия неопределенности типа 0/0 с использованием правила Лопиталя и дополнительного свойства функционала (80)

$$
\left.\frac{\partial Q_{\varepsilon}\{p(x)\}}{\partial \varepsilon}\right|_{\varepsilon=0}=-S_{0}\{p(x)\}
$$


которое можно получить с учетом равенства $(\partial / \partial \varepsilon) a^{\varepsilon}=a^{\varepsilon} \ln a$. В обоих случаях при любом $p(x)$ получаем

$$
S_{0}^{\mathrm{T}}\{p(x)\} \equiv S^{\mathrm{BGS}}\{p(x)\} .
$$

На основе общего представления (73) для семейства энтропийных функционалов $S_{\varepsilon}\{p(x)\}$ с $F_{\varepsilon}\{p(x)\}$ из (78) нетрудно показать, что для любых распределений $p(x)$ величина $S_{\varepsilon}$ является монотонно убьвающей функцией $\varepsilon$ по крайней мере на отрезке $[-1,1]$. В частности, отсюда следует, что $S_{\varepsilon}<S_{0}$ при $0<\varepsilon \leqslant 1$. Действительно,

$$
\frac{\partial S_{\varepsilon}\{p(x)\}}{\partial \varepsilon}=\int \frac{\partial F_{\varepsilon}[p(x)]}{\partial \varepsilon} d x<0,
$$

поскольку $(\partial / \partial \varepsilon) F_{\varepsilon}(a)=-a(\partial / \partial \varepsilon) \Psi_{\varepsilon}(a)(a>0)$. При этом производная

$$
\frac{\partial}{\partial \varepsilon} \Psi_{ \pm \varepsilon}(a)= \pm \frac{1}{\varepsilon}\left[\Psi_{0}(a)-\Psi_{ \pm \varepsilon}(a)\right]+\Psi_{0}(a) \Psi_{ \pm \varepsilon}(a)
$$

непрерывна по $\varepsilon$ и неотрицательна при всех $a$ и $-1 \leqslant \varepsilon \leqslant 1$, поскольку в точках $\varepsilon=$ $-1,0,1$ она имеет строго положительные значения при $a \neq 1$.

Проверка выполнения общих требований 1-3 из раздела 6 и наличие у вновь введенного энтропийного функционала $S_{\varepsilon}\{p(x)\}$ соответствующих свойств дает следующие результаты.

Выполнение требования 1 непосредственно следует из формул (73), (78), (85) и (87).

Требование 2а. Ограниченность снизу для $S_{\varepsilon}\{p(x)\}$ устанавливается для всех $\varepsilon>0$ с использованием $\varepsilon$-деформированной относительной энтропии (65) с выбором (66) для регуляризующей функции $\mu(x)$. Тогда первое слагаемое в квадратных скобках в правой части (91) с учетом первого из равенств (83) и того факта, что $\Delta x_{m} \sim m^{-1}$ при $m \rightarrow \infty$, для вырожденного распределения примет вид

$$
Q_{\varepsilon}\left\{p_{m}\left(x-x_{0}\right)\right\}\left(\Delta x_{m}\right)^{\varepsilon} \sim m^{\varepsilon}\left(m^{-1}\right)^{\varepsilon} \sim O(1) .
$$

Точного равенства правой части единице при любом $\varepsilon>0$ можно добиться, подобрав соответствуюший нормировочный множитель $c(\varepsilon)$ для величины $\Delta x_{m}(\varepsilon)=c(\varepsilon) \eta_{m}^{1 / 2}$. Подставляя результат в (91), находим одинаковый для всех $x_{0}$ и любого $\varepsilon>0$ нижний предел для обобщенной энтропии

$$
S_{\min }^{\varepsilon}\left\{\delta\left(x-x_{0}\right)\right\}=\lim _{m \rightarrow \infty} S_{\varepsilon}\left\{p_{m}\left(x-x_{0}\right)\right\}=0 .
$$

В случае $\varepsilon=0$ ситуация аналогична с тем лишь различием, что подлежаший компенсации вклад в $S_{0}$ имеет логарифмическую, а не степенную расходимость по $m$ (случай $\varepsilon<0$ рассматриваться не будет).

Требование 2б. Выполнение условий (89) и (90) обеспечивает ограниченность сверху для $S_{\varepsilon}\{p(x)\}$, поскольку для выпуклой в силу (87) функции $F_{\varepsilon}[p(x)]$ имеет место интегральное неравенство Иенсена

$$
\frac{\int F_{\varepsilon}[p(x)] d x}{\int d x} \leqslant F_{\varepsilon}\left[\frac{\int p(x) d x}{\int d x}\right] .
$$


Тогда, учитывая определения (2), (78) и условие нормировки из (1), получаем

$$
S_{\varepsilon}\{p(x)\} \leqslant S_{\varepsilon}^{\max },
$$

где

$$
S_{\varepsilon}^{\max }(W)=W F_{\varepsilon}\left(\frac{1}{W}\right)=-\Psi_{\varepsilon}\left(\frac{1}{W}\right)
$$

Нетрудно видеть, что абсолютный максимум (97) энтропии Цаллиса в полном соответствии с требованием $2 б$ достигается для равновероятного распределения (4), поскольку

$$
S_{\varepsilon}\left\{\frac{1}{W}\right\}=-\frac{1}{\varepsilon}\left[Q_{\varepsilon}\left\{\frac{1}{W}\right\}-1\right]=-\Psi_{\varepsilon}\left(\frac{1}{W}\right),
$$

где учтено второе из свойств (83) и определение (77).

В частности, при $\varepsilon=0$ выражения (96)-(98) приводят к ограниченности сверху равновесной канонической энтропии Клаузиуса (34) значением энтропии Больцмана

$$
S_{0}\{p(x)\} \leqslant S_{0}\left\{\frac{1}{W}\right\}=-\Psi_{0}\left(\frac{1}{W}\right)=\ln W
$$

которое легко получить непосредственно, положив в $(34) \bar{b}^{(0)}=0$ и $Z=W$.

Заметим, что при всех значениях $\varepsilon$ имеет место необходимое согласно (68) свойство

$$
\frac{\partial S_{\varepsilon}^{\max }(W)}{\partial W}=-\frac{\partial}{\partial W} \Psi_{\varepsilon}\left(\frac{1}{W}\right)=\left(\frac{1}{W}\right)^{\varepsilon+1}>0 .
$$

Требование 3. Требование (69) аддитивности энтропии для совместного мультипликативного распределения (70) в обшем случае $\varepsilon \neq 0$ не выполняется. Действительно, введенные выше функции (77) и (79) имеют следующие свойства для мультипликативных аргументов:

$$
\begin{gathered}
\Psi_{ \pm \varepsilon}\left(a_{1} a_{2}\right)=\Psi_{ \pm \varepsilon}\left(a_{1}\right)+\Psi_{ \pm \varepsilon}\left(a_{2}\right) \pm \varepsilon \Psi_{ \pm \varepsilon}\left(a_{1}\right) \Psi_{ \pm \varepsilon}\left(a_{2}\right) \\
P_{\varepsilon}\left(a_{1} a_{2}\right)=P_{\varepsilon}\left(a_{1}\right) P_{\varepsilon}\left(a_{2}\right), \quad P_{\varepsilon}(a)=a-\varepsilon F_{\varepsilon}(a)
\end{gathered}
$$

которые можно получить, используя равенства $\left(a_{1} a_{2}\right)^{\varepsilon}=a_{1}^{\varepsilon} a_{2}^{\varepsilon}$ и $a^{\varepsilon}=1+\Psi_{\varepsilon}(a)$. Тогда, очевидно, информационная функция (78) обладает свойством

$$
F_{\varepsilon}\left(a_{1} a_{2}\right)=a_{2} F_{\varepsilon}\left(a_{1}\right)+a_{1} F_{\varepsilon}\left(a_{2}\right)-\varepsilon F_{\varepsilon}\left(a_{1}\right) F_{\varepsilon}\left(a_{2}\right) .
$$

Подставляя $a_{1}=p\left(x_{1}\right)$ и $a_{2}=p\left(x_{2}\right)$, интегрируя по переменным $x_{1}$ и $x_{2}$ и учитывая условия нормировки (1) для обеих функций распределения, окончательно получаем для энтропии Цаллиса соотношение

$$
S_{\varepsilon}\{p(x)\}=S_{\varepsilon}\left\{p\left(x_{1}\right)\right\}+S_{\varepsilon}\left\{p\left(x_{2}\right)\right\}-\varepsilon S_{\varepsilon}\left\{p\left(x_{1}\right)\right\} S_{\varepsilon}\left\{p\left(x_{2}\right)\right\} .
$$

Этот же результат можно получить и непосредственно из выражения (91), если учесть, что $Q_{\varepsilon}=1-\varepsilon S_{\varepsilon}$, причем в силу первого из соотношений (101) имеем

$$
Q_{\varepsilon}\{p(x)\}=Q_{\varepsilon}\left\{p\left(x_{1}\right)\right\} Q_{\varepsilon}\left\{p\left(x_{2}\right)\right\} .
$$


Как видно из (103), требование аддитивности (69) удовлетворяется лишш для канонической энтропии Больцмана-Гиббса-Шеннона при $\varepsilon=0$. При $-1<\varepsilon<0$ соотношение (103) для энтропии Цаллиса приводит к свойству супераддитивности $\left(S_{12}>S_{1}+\right.$ $\left.S_{2}\right)$, а при $\varepsilon>0$ - к субаддитивности $\left(S_{12}<S_{1}+S_{2}\right)$. Подобные свойства могут, вообше говоря, иметь место для каких-либо статистически независимых объектов [31], в связи с чем требование (69) может быть ослаблено. Заметим, что для подобных объектов в соответствии со свойством (П.6) обобшенного распределения динамические величины (29) также удовлетворяют соотношению, в точности аналогичному (103),

$$
b(x)=b\left(x_{1}\right)+b\left(x_{2}\right)-\varepsilon b\left(x_{1}\right) b\left(x_{2}\right) .
$$

Ввиду неаддитивности энтропии Цаллиса $S_{\varepsilon}^{\mathrm{T}}\{p(x)\}$ и динамических величин $b(x)$ для статистически независимых объектов соответствующие обобщенные средние значения $A_{i}^{\mathrm{T}}\{p(x)\}$ также должны обладать этим свойством. Кроме того, их определение должно быть согласовано с видом функционала энтропии (91) так, чтобы выполнялось условие выпуклости (26) для функционала Лагранжа (22).

Наиболее подходяшим выбором в качестве сопровождаюшего распределения П $(a)$ в общем выражении (7) оказывается тогда ранее предложенньй в связи с распределением (56) выбор (59) или, в другой записи,

$$
\widetilde{\Pi}^{\mathrm{T}}(a) \equiv P_{\varepsilon}(a)=a^{\varepsilon+1},
$$

так что

$$
\bar{A}_{i}^{\mathrm{T}}=\int A_{i}(x) P_{\varepsilon}[p(x)] d x .
$$

Производные функционала (107) согласно (81) имеют в этом случае следуюший вид:

$$
\left[\bar{A}_{i}^{\mathrm{T}}\right]^{(n)}(a)=A_{i}(x) \frac{\partial^{n} P_{\varepsilon}(a)}{\partial a^{n}}=A_{i}(x) Q_{\varepsilon}^{(n)}(a), \quad a \equiv p(x),
$$

который при $n=1,2$ с точностью до множителя $A_{i}(x)$ совпадает с производными (88) для функционала энтропии $S_{\varepsilon}^{\mathrm{T}}$. В пределе $\varepsilon=0$ формула (108) правильно воспроизводит канонический результат (20).

Учитывая далее свойство (101) мультипликативности сопровождаюшего распределения (если этим свойством обладает основное распределение (70)), можно убедиться в неаддитивности средних значений $A_{i}^{\mathrm{T}}$ даже для аддитивной динамической величины $A_{i}(x)$. Действительно, имеем

$$
\bar{A}_{i}^{\mathrm{T}}\{p(x)\}=\bar{A}_{i}^{\mathrm{T}}\left\{p\left(x_{1}\right)\right\} Q_{\varepsilon}\left\{p\left(x_{2}\right)\right\}+\bar{A}_{i}^{\mathrm{T}}\left\{p\left(x_{2}\right)\right\} Q_{\varepsilon}\left\{p\left(x_{1}\right)\right\},
$$

и аддитивность средних значений восстанавливается лишь в каноническом пределе $\varepsilon=0$, когда согласно (81) $Q_{0} \equiv 1$. 
Нетрудно видеть, каким образом можно перейти к нормированным (и, как оказывается, аддитивным) средним значениям $A_{i}^{\mathrm{R}}\{p(x)\}^{12)}$. Для этого достаточно в качестве сопровождающего распределения выбрать нормированный вариант (106),

$$
\Pi^{\mathrm{R}}(a) \equiv \Pi^{\mathrm{T}}(a)=\frac{1}{Q_{\varepsilon}} P_{\varepsilon}=\frac{1}{Q_{\varepsilon}} a^{\varepsilon+1}, \quad a \equiv p(x)
$$

так что

$$
\bar{A}_{i}^{\mathrm{R}}=\frac{1}{Q_{\varepsilon}} \int A_{i}(x) P_{\varepsilon}[p(x)] d x=\frac{1}{Q_{\varepsilon}} \bar{A}_{i}^{\mathrm{T}} .
$$

Тогда, учитывая свойство (104) и деля обе части равенства (109) на величину (104), приходим к искомому соотношению аддитивности

$$
\bar{A}_{i}^{\mathrm{R}}\{p(x)\}=\bar{A}_{i}^{\mathrm{R}}\left\{p\left(x_{1}\right)\right\}+\bar{A}_{i}^{\mathrm{R}}\left\{p\left(x_{2}\right)\right\} .
$$

Трудности, однако, появляются при вычислении функциональных производных по $p(x)$ от средних значений (111) (см. ниже раздел 9).

\section{8. ОБОБШЕННАЯ ЭНТРОПИЯ РЕНЬИ И ХАР ДИ-ЛИТЛВУДА-ПОЙА}

Возможность устранить неаддитивность обобшенной энтропии состоит в переходе от энтропийного функционала Цаллиса $S_{\varepsilon}^{\mathrm{T}}\{p(x)\}$ к какому-либо иному функционалу, сохраняющему все свойства 1 и 2, но, кроме того, обладаюшему также свойством 3 (устранить неаддитивность динамических величин (105) таким способом все равно не удается, поскольку она обусловлена свойством (П.6) обобщенного распределения (56)). С этой точки зрения наиболее подходяшим представляется энтропийный функционал $S_{\varepsilon}^{\mathrm{R}}\{p(x)\}$, введенный Реньи [16] (для дискретного случая) задолго до работы Цаллиca $[17]$.

Функционалы $S_{\varepsilon}^{\mathrm{R}}$ и $S_{\varepsilon}^{\mathrm{T}}$ связаны друг с другом прямым $R_{\varepsilon}^{ \pm}$и обратным $\left[R_{\varepsilon}^{ \pm}\right]^{-1}$ преобразованиями, которые естественно назвать преобразованиями Реньи. Преобразование $R_{\varepsilon}^{ \pm}$ставит в соответствие любой вешественной величине $f_{ \pm}$ее (также вешественный) "реньи-образ" $f_{ \pm}^{\mathrm{R}}$, имеюший тот же знак, что и $f_{ \pm}$,

$$
f_{ \pm}^{\mathrm{R}} \equiv \widehat{R}_{\varepsilon}^{ \pm} f_{ \pm}=\mp \frac{1}{\varepsilon} \ln \left[1 \mp \varepsilon f_{ \pm}\right], \quad \widehat{R}_{0}^{ \pm}=1
$$

В формулах (113) верхний или нижний знаки выбираются соответственно для $f_{+}>0$ или $f_{-}>0$, а условием применимости преобразования (110) служит неравенство $1 \pm$ $\varepsilon f_{ \pm}>0$.

Преобразование (113) является нелинейным, невырожденным,

$$
f_{ \pm}=\left[\widehat{R}_{\varepsilon}^{ \pm}\right]^{-1} f_{ \pm}^{\mathrm{R}}=\mp \frac{1}{\varepsilon}\left[\exp \left(\mp \varepsilon f_{ \pm}^{\mathrm{R}}\right)-1\right]
$$

\footnotetext{
12) Верхний индекс R (Реньи) введен пока формально. В разделе 8 будет показано, что он действительно относится к выбору энтропийного функционала и средних значений по Реньи, а не по Цаллису.
} 
и сжимающим

$$
f_{ \pm}^{\mathrm{R}} \leqslant f_{ \pm} \quad \text { при } \quad \varepsilon f_{ \pm} \neq 0, \quad f_{ \pm}^{\mathrm{R}}=f_{ \pm} \quad \text { при } \quad \varepsilon f_{ \pm}=0,
$$

поскольку для любого положительного значения $x$ имеем $\ln x \leqslant x-1$. Заметим, что при малых значениях произведения $\varepsilon f_{ \pm}$имеем $f_{ \pm}^{\mathrm{R}} \approx f_{ \pm}$.

Полезность преобразования Реньи состоит в том, что оно переводит информационную функцию Цаллиса $F_{\varepsilon}$ в каноническую $F_{0}$ без перехода к пределу $\varepsilon \rightarrow 0$ (при любом конечном значении $\varepsilon$ и любом $a>0$ ), так как

$$
\Psi_{0}(a)=\widehat{R}_{\varepsilon}^{-} \Psi_{\varepsilon}(a) .
$$

Применительно же к самой энтропии Цаллиса это не так и

$$
S_{0}^{\mathrm{BGS}} \neq \widehat{R}_{\varepsilon}^{-} S_{\varepsilon}^{\mathrm{T}} .
$$

Тем не менее целесообразно определить энтропию Реньи как реньи-образ энтропии Цаллиса

$$
S_{\varepsilon}^{\mathrm{R}}\{p(x)\}=\widehat{R}_{\varepsilon}^{-} S_{\varepsilon}^{\mathrm{T}}\{p(x)\},
$$

откуда в силу (115) имеем

$$
S_{\varepsilon}^{\mathrm{R}}\{p(x)\} \leqslant S_{\varepsilon}^{\mathrm{T}}\{p(x)\} .
$$

С учетом явного вида $S_{\varepsilon}^{\mathrm{T}}$ из $(91)$ и $R_{\varepsilon}^{-}$из $(113)$ находим

$$
S_{\varepsilon}^{\mathrm{R}}\{p(x)\}=-\frac{1}{\varepsilon} \ln \left[1-\varepsilon S_{\varepsilon}^{\mathrm{T}}\{p(x)\}\right]=-\frac{1}{\varepsilon} \ln Q_{\varepsilon}\{p(x)\} .
$$

Благодаря положительности функционала $Q_{\varepsilon}\{p(x)\}$ из (80) выражение (119) имеет смысл при любых $p(x)$ и $\varepsilon$.

Аналогично при любых $p(x)$ и $\varepsilon$ можно определить еще один энтропийный функционал, ранее фактически введенный Харди, Литлвудом и Пойа [32],

$$
S_{\varepsilon}^{\mathrm{HLP}}\{p(x)\}=\left[1-\varepsilon S_{\varepsilon}^{\mathrm{T}}\{p(x)\}\right]^{-1 / \varepsilon}=\left[Q_{\varepsilon}\{p(x)\}\right]^{-1 / \varepsilon},
$$

так что

$$
S_{\varepsilon}^{\mathrm{HLP}}\{p(x)\}=\exp \left[S_{\varepsilon}^{\mathrm{R}}\{p(x)\}\right] .
$$

Обратные к $(119)$ и $(120)$ выражения для $S_{\varepsilon}^{\mathrm{T}}$ через $S_{\varepsilon}^{\mathrm{R}}$ и $S_{\varepsilon}^{\mathrm{HLP}}$ имеют вид

$$
S_{\varepsilon}^{\mathrm{T}}\{p(x)\}=-\frac{1}{\varepsilon}\left[\exp \left(-\varepsilon S_{\varepsilon}^{\mathrm{R}}\{p(x)\}\right)-1\right]=-\frac{1}{\varepsilon}\left[\left(S_{\varepsilon}^{\mathrm{HLP}}\{p(x)\}\right)^{-\varepsilon}-1\right],
$$

или в сокрашенной форме

$$
S_{\varepsilon}^{\mathrm{T}}\{p(x)\}=\left[\widehat{R}_{\varepsilon}^{+}\right]^{-1} S_{\varepsilon}^{\mathrm{R}}\{p(x)\}=\Psi_{-\varepsilon}\left[S_{\varepsilon}^{\mathrm{HLP}}\{p(x)\}\right] .
$$

В пределе $\varepsilon=0$ с учетом свойств $\widehat{R}_{0}^{( \pm)}=\left[\widehat{R}_{0}^{( \pm)}\right]^{-1}=1$ из $(113)$ и $\Psi_{0}(a)=\ln a$ из $(116)$ имеем

$$
S_{0}^{\mathrm{T}}\{p(x)\}=S_{0}^{\mathrm{R}}\{p(x)\}=\ln S_{0}^{\mathrm{HLP}}\{p(x)\}=S_{0}^{\mathrm{BGS}}\{p(x)\} .
$$


Заметим, что функционалы $S_{\varepsilon}^{\mathrm{R}}$ и $S_{\varepsilon}^{\mathrm{HLP}}$ в отличие от $S_{\varepsilon}^{\mathrm{T}}$ не могут быть представлены в виде (73) с помощью какой-либо информационной функции: эти функционалы выражаются через $Q_{\varepsilon}$ нелинейным образом, тогда как $S_{\varepsilon}^{\mathrm{T}}-$ линейным.

Проверим теперь выполнение требований 1-3 (см. выше раздел 6) энтропийной аксиоматики применительно ко вновь введенным видам обобшенной энтропии (или, что то же, наличие у нее свойств 1-3). Свойство 1 следует непосредственно из определений (119) и $(120)$, причем, опуская аргумент $p(x)$, имеем

$$
\begin{aligned}
\left(S_{\varepsilon}^{\mathrm{R}}\right)^{\prime} & =\frac{1}{Q_{\varepsilon}}\left(S_{\varepsilon}^{\mathrm{T}}\right)^{\prime}, & \left(S_{\varepsilon}^{\mathrm{R}}\right)^{\prime \prime} & =\frac{1}{Q_{\varepsilon}}\left\{\frac{\varepsilon}{Q_{\varepsilon}}\left[\left(S_{\varepsilon}^{\mathrm{T}}\right)^{\prime}\right]^{2}+\left(S_{\varepsilon}^{\mathrm{T}}\right)^{\prime \prime}\right\}, \\
\left(S_{\varepsilon}^{\mathrm{HLP}}\right)^{\prime} & =Q_{\varepsilon}^{-\frac{\varepsilon+1}{\varepsilon}}\left(S_{\varepsilon}^{\mathrm{T}}\right)^{\prime}, & \left(S_{\varepsilon}^{\mathrm{HLP}}\right)^{\prime \prime} & =Q_{\varepsilon}^{-\frac{\varepsilon+1}{\varepsilon}}\left\{\frac{1+\varepsilon}{Q_{\varepsilon}}\left[\left(S_{\varepsilon}^{\mathrm{T}}\right)^{\prime}\right]^{2}+\left(S_{\varepsilon}^{\mathrm{T}}\right)^{\prime \prime}\right\},
\end{aligned}
$$

где производные $\left(S_{\varepsilon}^{\mathrm{T}}\right)^{(n)}(n=1,2)$ даются выражениями $(88)$.

Свойства 2а и $2 б$ также следуют из свойств (83) функционала $Q_{\varepsilon}$ и рассуждений, приведенных перед формулой (95). Абсолютный минимум для (119) и (120) имеет вид соответственно

$$
\begin{gathered}
{\left[S_{\varepsilon}^{\mathrm{R}}\right]^{\mathrm{min}}=S_{\varepsilon}^{\mathrm{R}}\left\{\delta\left(x-x_{0}\right)\right\}=\lim _{m \rightarrow \infty} S_{\varepsilon}^{\mathrm{R}}\left\{p_{m}\left(x-x_{0}\right)\right\}=0,} \\
{\left[S_{\varepsilon}^{\mathrm{HLP}}\right]^{\min }=S_{\varepsilon}^{\mathrm{HLP}}\left\{\delta\left(x-x_{0}\right)\right\}=\lim _{m \rightarrow \infty} S_{\varepsilon}^{\mathrm{HLP}}\left\{p_{m}\left(x-x_{0}\right)\right\}=1,}
\end{gathered}
$$

а абсолютный максимум -

$$
\begin{aligned}
{\left[S_{\varepsilon}^{\mathrm{R}}\right]^{\mathrm{max}} } & =S_{\varepsilon}^{\mathrm{R}}\left\{\frac{1}{W}\right\}=-\frac{1}{\varepsilon} \ln Q_{\varepsilon}\left\{\frac{1}{W}\right\}=\ln W, \\
{\left[S_{\varepsilon}^{\mathrm{HLP}}\right]^{\max } } & =S_{\varepsilon}^{\mathrm{HLP}}\left\{\frac{1}{W}\right\}=\left[Q_{\varepsilon}\left\{\frac{1}{W}\right\}\right]^{-1 / \varepsilon}=W .
\end{aligned}
$$

Очевидно, в случаях (127) и (128) правые части, как и требуется, не зависят от $x_{0}$ и $\varepsilon$, а в случаях (129) и (130) также не зависят от $\varepsilon$ и в соответствии с (68) имеют положительную производную по $W$.

Что касается свойства 3 , то, используя формулу (104), нетрудно получить для энтропий (119) и (120) соответственно

$$
\begin{aligned}
S_{\varepsilon}^{\mathrm{R}}\{p(x)\} & =S_{\varepsilon}^{\mathrm{R}}\left\{p\left(x_{1}\right)\right\}+S_{\varepsilon}^{\mathrm{R}}\left\{p\left(x_{2}\right)\right\}, \\
S_{\varepsilon}^{\mathrm{HLP}}\{p(x)\} & =S_{\varepsilon}^{\mathrm{HLP}}\left\{p\left(x_{1}\right)\right\} S_{\varepsilon}^{\mathrm{HLP}}\left\{p\left(x_{2}\right)\right\} .
\end{aligned}
$$

Таким образом, по отношению к требованию 3 функционалы $S_{\varepsilon}^{\mathrm{R}}$ и $S_{\varepsilon}^{\mathrm{HLP}}$ при любых $\varepsilon$ и любых $p(x)$ представляют собой предельные случаи: адлитивности в случае (131) и мультипликативности в случае (132). Функционал $S_{\varepsilon}^{\mathrm{T}}$ согласно (103) в этом смысле представляет собой промежуточный случай, который сводится к аддитивности при $\varepsilon=0$ и к мультипликативности при $\varepsilon \rightarrow-\infty$.

Таким образом, по совокупности свойств 1-3 энтропийный функционал Реньи $S_{\varepsilon}^{\mathrm{R}}$ при любых $\varepsilon$ в точности совпадает с каноническим энтропийным функционалом Больцмана-Гиббса-Шеннона $S_{0}^{\mathrm{BGS}}$ и с этой точки зрения является, по-видимому, наиболее 
предпочтительным из всех рассмотренных. Близким к $S_{0}^{\text {BGS }}$ (во всяком случае, в пределе малых $\varepsilon$ ) оказывается также энтропийный функционал Цаллиса $S_{\varepsilon}^{\mathrm{T}}$. Что касается энтропийного функционала $S_{\varepsilon}^{\mathrm{HLP}}$, то его свойства (особенно 3 ) радикально расходятся со свойствами $S_{0}^{\mathrm{BGS}}$. Тем не менее не исключено, что и функционал $S_{\varepsilon}^{\mathrm{HLP}}$ может быть применим к некоторым вероятностным объектам, для которых требование 3 следует ослабить или модифицировать (см. раздел 13).

Благодаря аддитивности энтропии Реньи $S_{\varepsilon}^{\mathrm{R}}$ вопрос о выборе соответствующих ей обобщенных средних значений решается однозначно (для энтропии Харди-ЛитлвудаПойа этот вопрос не имеет столь однозначного решения и требует дополнительного анализа, который здесь не приводится). В этом качестве должны быть использованы введенные формулой (111) аддитивные средние $A_{k}^{\mathrm{R}}$, или в явном виде

$$
\bar{A}_{i}^{\mathrm{R}}\{p(x)\}=\left[Q_{\varepsilon}\{p(x)\}\right]^{-1} \bar{A}_{i}^{\mathrm{T}}\{p(x)\} .
$$

Дифференцируя (133) и опуская для краткости аргумент $p(x)$ и индекс $\varepsilon$ у $Q_{\varepsilon}$, находим для первой функциональной производной

$$
\left(\bar{A}_{i}^{\mathrm{R}}\right)^{\prime}=\frac{1}{Q}\left(\bar{A}_{i}^{\mathrm{T}}\right)^{\prime}-\frac{1}{Q^{2}} Q^{\prime} \bar{A}_{i}^{\mathrm{T}}=\frac{Q^{\prime}}{Q} \Delta A_{i}^{\mathrm{R}},
$$

где

$$
\Delta A_{i}^{\mathrm{R}} \equiv A_{i}(x)-\bar{A}_{i}^{\mathrm{R}}
$$

- флуктуация динамической величины $A_{i}(x)$ относительно нормированного среднего значения, определенного в (133). Соответственно для второй функциональной производной имеем

$$
\left(\bar{A}_{i}^{\mathrm{R}}\right)^{\prime \prime}=-\frac{1}{Q^{2}}\left[2\left(Q^{\prime}\right)^{2}-Q Q^{\prime \prime}\right] \Delta A_{i}^{\mathrm{R}} .
$$

Сравнение с соотношениями (125) показьвает, что если выражение (134) линейно содержит величину $\left(S_{\varepsilon}^{\mathrm{R}}\right)^{\prime}$, то выражение $(136)$ полностью рассогласовано с $\left(S_{\varepsilon}^{\mathrm{R}}\right)^{\prime \prime}$. Кроме того, пределы обоих выражений (134) и (136) при $\varepsilon=0$ не совпадают с каноническими значениями (20).

\section{9. ВАРИАЦИОННАЯ ПРОЦЕДУРА ДЛЯ ОБОБЩЕННОЙ ЭНТРОПИИ И НЕЛИНЕЙНЫХ СРЕДНИХ ЗНАЧЕНИЙ}

Используем теперь полученные в разделах 7 и 8 результаты для проведения вариационной процедуры, описанной в разделе 3 . Рассмотрим последовательно случаи энтропий Цаллиса и Реньи.

9.1. Энтропия Цаллиса и ненормированные средние значения. Подставим в функционал Лагранжа (22) выражения (91) для энтропии Цаллиса и (107) для средних значений, а затем дважды продифференшируем $\mathcal{L}_{\varepsilon}^{\mathrm{T}}$. Учитывая соотношения (88) и (108), а также (81) и определение (79), получим (опуская для краткости аргументы $x$ и $p(x)$ ) вместо (24) и (25) формулы

$$
\begin{aligned}
\left(\mathcal{L}_{\varepsilon}^{\mathrm{T}}\right)^{\prime} & =-\frac{1}{\varepsilon}\left\{Q_{\varepsilon}^{\prime}[1-\varepsilon b]-\left(1-\varepsilon \alpha_{0}\right)\right\}=-\frac{1}{\varepsilon}\left\{(\varepsilon+1) p^{\varepsilon}[1-\varepsilon b]-\left(1-\varepsilon \alpha_{0}\right)\right\}, \\
\left(\mathcal{L}_{\varepsilon}^{\mathrm{T}}\right)^{\prime \prime} & =-\frac{1}{\varepsilon} Q_{\varepsilon}^{\prime \prime}[1-\varepsilon b]=-(\varepsilon+1) p^{\varepsilon-1}[1-\varepsilon b] .
\end{aligned}
$$


Из (138) сразу следует, что если выполнены условия $(90)$ и $(60)$, то $\left(\mathcal{L}_{\varepsilon}^{\mathrm{T}}\right)^{\prime \prime}<0$ и функционал $\mathcal{L}_{\varepsilon}^{\mathrm{T}}$ является выпуклым. Подставляя далее $(137)$ в уравнение экстремума $(23)$, получим для равновесного распределения Цаллиса $p_{\varepsilon}^{\mathrm{T}}(x)$ в точности выражение (56). Изменится лишш связь между $Z_{\varepsilon}^{\mathrm{T}}$ и $\alpha_{0}$,

$$
Z_{\varepsilon}^{\mathrm{T}}=\left(\frac{1-\varepsilon \alpha_{0}}{1+\varepsilon}\right)^{-1 / \varepsilon}, \quad Z_{0}^{\mathrm{T}}=e^{\alpha_{0}+1}=Z_{0}^{\mathrm{BGS}} .
$$

Таким образом, несмотря на нелинейность по $p(x)$ определения ненормированных обобщенных средних значений (107), пропорциональность их производных (108) по $p(x)$ производным того же порядка (88) для энтропии Цаллиса приводит к равновесному решению вида (56), характерному, вообще говоря, для случая линейного определения средних значений и произвольного (но выпуклого) функционала для энтропии (свойства этого решения подробно рассмотрены в разделе 5 , а также в приложении).

9.2. Энтропия Реньи и нормированные средние значения. Подставим далее в функционал Лагранжа (22) выражения (119) для энтропии Реньи и (111) для средних значений, а затем также дважды продифференцируем функционал $\mathcal{L}_{\varepsilon}^{\mathrm{R}}$. Учитывая соотношения (125), (134) и (136), получим тогда (вновь опуская аргументы $x$ и $p(x))$

$$
\begin{aligned}
\left(\mathcal{L}_{\varepsilon}^{\mathrm{R}}\right)^{\prime} & =-\frac{1}{\varepsilon Q_{\varepsilon}}\left\{Q_{\varepsilon}^{\prime}\left[1-\Delta b^{\mathrm{R}}\right]-\left(1-\varepsilon Q_{\varepsilon} \alpha_{0}\right)\right\}, \\
\left(\mathcal{L}_{\varepsilon}^{\mathrm{R}}\right)^{\prime \prime} & =\frac{1}{\varepsilon Q_{\varepsilon}^{2}}\left\{\left[\left(Q_{\varepsilon}^{\prime}-1\right)^{2}-Q_{\varepsilon} Q_{\varepsilon}^{\prime \prime}\right]-\varepsilon\left[2 Q_{\varepsilon}^{\prime}-Q_{\varepsilon} Q_{\varepsilon}^{\prime \prime}\right] \Delta b^{\mathrm{R}}\right\},
\end{aligned}
$$

где $\Delta b^{\mathrm{R}}(x)=b^{\mathrm{R}}(x)-\bar{b}^{\mathrm{R}}$. Из выражения (141) видно, что рассогласование (отсутствие пропорциональности) производных (125) энтропии Реньи и средних (136) во втором порядке не позволяет получить определенное суждение о знаке $\left(\mathcal{L}_{\varepsilon}^{\mathrm{R}}\right)^{\prime \prime}$ (и, следовательно, о выпуклости $\mathcal{L}_{\varepsilon}^{\mathrm{R}}$ ).

Что касается равновесного решения уравнения экстремума $(23)$ для $\mathcal{L}_{\varepsilon}^{\mathrm{R}}$, то оно формально существует и имеет вид (56) с одним (но весьма важным) отличием - заменой $b \rightarrow \Delta b^{\mathrm{R}}$. Несмотря на это, в пределе $\varepsilon=0$ решение $p_{\varepsilon}^{\mathrm{R}}$ переходит в $p_{0}^{\mathrm{BGS}}$, поскольку не зависящий от $x$ множитель $\exp \left(\sum \alpha_{i} A_{i}^{\mathrm{R}}\right)$ сократится.

Заметим, однако, что статистический интеграл $Z_{\varepsilon}^{\mathrm{R}}$ из (44) теряет свое производящее свойство, поскольку

$$
\frac{\partial}{\partial \beta} Z_{\varepsilon}^{\mathrm{R}}(\beta)=\overline{\Delta b^{\mathrm{R}}(x)} \equiv 0
$$

Кроме того, для $\left(\mathcal{L}_{\varepsilon}^{\mathrm{R}}\right)^{\prime \prime}$ характерно отсутствие знакоопределенности (даже для равновесного решения $p_{\varepsilon}^{\mathrm{R}}$ ), что затрудняет выяснение характера этого решения (минимум, максимум или точка перегиба для функционала Лагранжа). В сочетании с соотношением (142) целесообразность использования решения $p_{\varepsilon}^{\mathrm{R}}$ не представляется достаточно убедительной ${ }^{13)}$.

13) В работе [33] решение $p_{\varepsilon}^{\mathrm{R}}$ используется в асимптотическом пределе больших $x$ на ограниченном как сверху, так и снизу интервале изменения исходной случайной переменной $x$. Для этого решения переопределяется нормировочный множитель $Z_{\varepsilon}^{\mathrm{R}}$. 
В связи с этим дальнейшая программа, реализуемая в разделах 11 и 12 , состоит в построении двух вариантов обобшенной РСМ (для частного случая одномерного идеального газа) как для энтропии Цаллиса, так и для энтропии Реньи на основе только равновесного решения Цаллиса $p_{\varepsilon}^{\mathrm{T}}$. Этот путь, разумеется, также не является вполне последовательным и приводит к необходимости процедуры самосогласования двух вариантов PCM (см. формулу (206) в разделе 12). Перед этим в разделе 10 будет в общем виде показана применимость соотношений Гиббса-Гельмгольца не только для канонической (см. раздел 4), но и для обобщенной РСМ (в данном случае только для варианта Цаллиса), построенной на основе вариационного принципа для информационной энтропии (см. раздел 3$)$.

\section{0. ОБОБШЕННЫЕ СООТНОШЕНИЯ ГИББСА-ГЕЛЬМГОЛЬЦА}

В разделе 3 была сформулирована вариационная процедура нахождения равновесного распределения для наиболее общего случая, когда нелинейными функционалами являются как энтропия $S$, так и все средние значения $A_{i}$. Для дальнейшего построения на этой основе обобшенной РСМ значительный интерес представляют соотношения Гиббса-Гельмгольца, аналогичные имеюшим место в канонической РСМ (формулы (36)-(38)). Покажем, что применимость этих соотношений действительно следует непосредственно из указанного выше вариационного принципа для обобщенной энтропии и фиксированных средних значений.

Заметим прежде всего, что решением уравнения экстремума (23) (при выполнении условия (26)) является нормированное равновесное распределение $p^{\mathrm{eq}}\left(x ;\left\{\alpha_{i}^{\mathrm{eq}}\right\}\right)$, зависящее от равновесных множителей Лагранжа $\left\{\alpha_{i}^{\text {eq }}\right\}(i \geqslant 1)$ как от параметров. При этом множитель $\alpha_{0}^{\text {eq }}=\alpha_{0}\left\{\alpha_{i}^{\text {eq }}\right\}$ определяется из условия нормировки (1) и обычно выражается через статистический интеграл (например, в виде (139)). Остальные множители $\alpha_{i}^{\mathrm{eq}}=\alpha_{i}\left\{A_{k}\right\}$ находятся из условий равновесия (5), где $A_{i}$ - равновесные значения средних величин $\bar{A}_{i}$. Ясно, что формально можно рассматривать и обратную зависимость $A_{i}=A_{i}\left\{\alpha_{k}^{\text {eq }}\right\}$, причем обе зависимости представляют собой сложную систему нелинейных уравнений.

Таким образом, обобшенная равновесная энтропия $S_{\text {eq }}\left[p^{\mathrm{eq}}\left(x ;\left\{\alpha_{i}^{\mathrm{eq}}\right\}\right)\right]$ по построению является явной функцией множителей Лагранжа $\left\{\alpha_{i}^{\mathrm{eq}}\right\}$ или неявной функцией величин $\left\{A_{i}\right\}$. Поэтому для энтропии представляют интерес наборы соответствующих производных $\partial S / \partial \alpha_{i}^{\mathrm{eq}}$ и $\partial S / \partial A_{i}$. Опуская для краткости аргументы $x$ и $\left\{\alpha_{i}^{\mathrm{eq}}\right\}$ у функции $p^{\mathrm{eq}}\left(x ;\left\{\alpha_{i}^{\mathrm{eq}}\right\}\right)$, а также верхний индекс еq у $\left\{\alpha_{i}^{\mathrm{eq}}\right\}$, имеем тогда

$$
\frac{\partial S_{\mathrm{eq}}}{\partial \alpha_{k}}=\int d x S^{\prime}\left[p^{\mathrm{eq}}\right] \frac{\partial p^{\mathrm{eq}}}{\partial \alpha_{k}}, \quad \frac{\partial \bar{A}_{m}}{\partial \alpha_{k}}=\int d x \bar{A}_{m}^{\prime}\left[p^{\mathrm{eq}}\right] \frac{\partial p^{\mathrm{eq}}}{\partial \alpha_{k}}
$$

при всех значениях $k \geqslant 1$. Здесь штрих, как и ранее, обозначает функциональную производную $S_{\text {eq }}$ и $\bar{A}_{i}$ по распределению $p^{\text {eq }}$. С учетом условия экстремума $(23)$ и опреде-

2 Теоретическая и математическая физика, т. 135, № 1, 2003 г. 
ления (24) получаем

$$
\begin{aligned}
\frac{\partial S_{\mathrm{eq}}}{\partial \alpha_{k}}-\sum_{m \geqslant 1} \alpha_{m} \frac{\partial A_{m}}{\partial \alpha_{k}} & =\int d x\left\{S^{\prime}\left[p^{\mathrm{eq}}\right]-\sum_{m \geqslant 1} \alpha_{m} A_{m}^{\prime}\left[p^{\mathrm{eq}}\right]\right\} \frac{\partial p^{\mathrm{eq}}}{\partial \alpha_{k}}= \\
& =\int d x\left\{\mathcal{L}^{\prime}\left[p^{\mathrm{eq}}\right]+\alpha_{0}\right\} \frac{\partial p^{\mathrm{eq}}}{\partial \alpha_{k}}=\alpha_{0} \int d x \frac{\partial p^{\mathrm{eq}}}{\partial \alpha_{k}}=0,
\end{aligned}
$$

где учтено, что согласно условию нормировки (1) имеет место равенство

$$
\frac{\partial}{\partial \alpha_{k}} \int d x p^{\mathrm{eq}}=\int d x \frac{\partial p^{\mathrm{eq}}}{\partial \alpha_{k}}=0 .
$$

Из (144) следует, что

$$
\frac{\partial S_{\mathrm{eq}}}{\partial \alpha_{k}}=\sum_{m \geqslant 1} \frac{\partial S_{\mathrm{eq}}}{\partial \alpha_{m}} \frac{\partial \alpha_{m}}{\partial A_{k}}=\sum_{m \geqslant 1} \sum_{l \geqslant 1} \alpha_{l} \frac{\partial A_{l}}{\partial \alpha_{m}} \frac{\partial \alpha_{m}}{\partial A_{k}} .
$$

Учитывая далее очевидное равенство

$$
\sum_{m \geqslant 1} \frac{\partial A_{l}}{\partial \alpha_{m}} \frac{\partial \alpha_{m}}{\partial A_{k}}=\frac{\partial A_{l}}{\partial A_{k}}=\delta_{l k},
$$

приходим к производяшему свойству для энтропии

$$
\frac{\partial S_{\mathrm{eq}}\left\{A_{i}\right\}}{\partial A_{k}}=\alpha_{k} .
$$

Отсюда видно, что величины $A_{i}$ являются "естественными" переменными для энтропии $S_{\text {eq }}$, поскольку знание функции $S_{\text {eq }}\left\{A_{i}\right\}$ позволяет избежать решения сложной системы уравнений для отыскания $\left\{\alpha_{k}\right\}$.

Свойство (147) дает возможность записать $S_{\text {eq }}\left\{A_{i}\right\}$ также в виде

$$
S_{\text {eq }}\left\{A_{i}\right\}=\Phi\left\{\alpha_{i}\right\}+\sum_{k \geqslant 1} \alpha_{k} A_{k},
$$

или в соответствии с соотношением (30) в виде

$$
S\left\{A_{i}\right\}-\Phi\left\{\alpha_{i}\right\}=-\bar{b} .
$$

Входящая в (148) и (149) величина $\Phi\left\{\alpha_{i}\right\}$ называется обобщенной функиией МасьеПланка. По определению она должна обладать аналогичным (147) производящим свойством по переменным $\alpha_{k}$ :

$$
\frac{\partial \Phi\left\{\alpha_{i}\right\}}{\partial \alpha_{k}}=-A_{k} .
$$

Соотношение (148) (или эквивалентное ему (149)) представляет собой взаимное $n p e-$ образование Лежандра между переменными $\left\{A_{i}\right\}$ и $\left\{\alpha_{i}\right\}$. В его справедливости легко 
убедиться, беря полные дифференциалы левой и правой частей равенства (148) и учитывая свойства (147) и (150). Эти свойства совместно с равенством (148) составляют содержание обобшенных соотношений Гиббса-Гельмгольца и дают основание называть величины $S_{\text {еq }}$ и характеристическими функииями в соответствуюших переменных. Вид этих функций (при заданной зависимости исходных динамических переменных $E(x))$ зависит от вида используемого функционала $S\{p(x)\}$. Целесообразность использования этих функций зависит от постановки исходной задачи описания вероятностного объекта: фиксирования средних значений экстенсивных величин $\left\{A_{i}\right\}$ или сопряженных им интесивных величин $\left\{\alpha_{i}\right\}$. Подчеркнем, что все введенные выше понятия и соотношения обусловлены вариационной процедурой нахождения равновесного распределения и сохраняют применимость для весьма общего случая неканонической РСМ.

\section{1. ОБОБЩЕННАЯ РСМ ДЛЯ ОДНОМЕРНОГО ИДЕАЛЬНОГО ГАЗА. ОБШИЕ СООТНОШЕНИЯ}

Рассмотрим простейший частный случай изложенной выше обшей схемы, когда пространство состояний $X$ вероятностного объекта является одномерным $(d=1)$, а набор динамических величин - однокомпонентным,

$$
\alpha_{1} \equiv \beta, \quad A_{1}(x) \equiv E(x), \quad b(x)=-\beta E(x) .
$$

Следуя термодинамической аналогии, такой объект можно считать “идеальным газом" с одной степенью свободы $x$, обладающим “энергией” $E(x)$ и “обратной температурой” $\beta$ или “абсолютной температурой" $T=1 / \beta$ (в энергетических единицах).

Ограничимся далее случаем, когда положительная энергия $E(x) \geqslant 0$ для также положительных значений $x \geqslant 0$ описывается монотонно возрастающей степенной функцией. Будем считать, что $E(x)$ не имеет ограничения сверху, так что $\beta$ или $T$ также могут принимать лишь положительные значения $(\beta \geqslant 0, T \geqslant 0)$. Тогда

$$
-b(x)=\beta E(x)=\beta \zeta x^{h} \equiv\left[\frac{x}{x_{\mathrm{c}}(\beta)}\right]^{h} \geqslant 0,
$$

где

$$
x_{\mathrm{c}}(\beta) \equiv(\beta \zeta)^{-1 / h}, \quad x_{\mathrm{c}}(\beta) \geqslant 0,
$$

причем “динамический” показатель $h>0$ положителен и безразмерен, а коэффициент $\zeta>0$ также положителен, но имеет размерность энергии, так что произведение $\beta \zeta$ оказывается безразмерным.

В случае выбора $b(x)$ в виде $(152)$ (при этом в силу $(61) \varepsilon \geqslant 0$ ) обобшенное ненормированное распределение (56) принимает вид

$$
\tilde{p}_{\varepsilon}(x)=\left\{1+\varepsilon\left[\frac{x}{x_{\mathrm{c}}(\beta)}\right]^{h}\right\}^{-1 / \varepsilon},
$$

что при $\varepsilon=0$ переходит в

$$
\tilde{p}_{0}(x)=\exp \left\{-\left[\frac{x}{x_{\mathrm{c}}(\beta)}\right]^{h}\right\}
$$


Весьма существенно, что распределения (154) и (155) имеют качественно различную асимптотику при больших значениях $x$. Объединяя эти выражения с формулами (П.23) и (П.24), имеем в каноническом случае

$$
\tilde{p}_{0}(x) \sim \exp \left\{-\left[\frac{x}{x_{\mathrm{c}}(\beta)}\right]^{h}\right\},
$$

а в обобщенном случае

$$
\tilde{p}_{\varepsilon}(x) \sim \varepsilon^{-1 / \varepsilon}\left[\frac{x}{x_{\mathrm{c}}(\beta)}\right]^{-s_{\varepsilon}(h)} .
$$

Здесь $s_{\varepsilon}(h)=h / \varepsilon$ - всегда положительная величина, причем согласно полученному ниже условию (168) для "кинематического" показателя $\varepsilon$ эту величину можно представить в виде

$$
s_{\varepsilon}(h)=1+\tilde{\delta}_{\varepsilon}(h), \quad \tilde{\delta}_{\varepsilon}(h) \equiv \frac{h}{\varepsilon}-1>0 .
$$

Очевидно, что в выражениях (157) и (158) предельный переход к случаю $\varepsilon=0$ уже невозможен, хотя, вообще говоря, показатели $\tilde{\delta}_{\varepsilon}$ и $s_{\varepsilon}$ могут быть сколь угодно велики и, следовательно, распределение (157) при больших $x$ может быть сколь угодно близко к (156).

Заметим, однако, что получить асимптотику вида (157) в случае исходного канонического распределения (155) возможно лиш при весьма искусственном предположении о логарифмической зависимости энергии $E(x)=s \ln \left[x / x_{\mathrm{c}}(\beta)\right]$ от переменной состояния $x$. Значительно более естественным представляется для этого переход к обобщенному распределению (154) со степенной зависимостью энергии (152).

Степенная асимптотика (157) при $\varepsilon>0$ и $x \rightarrow \infty$ указывает на принадлежность одномерного распределения $p_{\varepsilon}(x)$ к классу устойчивых распределений [5], [28], к которым применима центральная предельная теорема. Если применить к распределению $p_{\varepsilon}(x)$ масштабное преобразование

$$
p_{\varepsilon}^{(N)}(x)=N^{-1 / \tilde{\delta}_{\varepsilon}} p_{\varepsilon}\left(x N^{-1 / \tilde{\delta}_{\varepsilon}}\right),
$$

то полученное распределение $p_{\varepsilon}^{(N)}(x)$ описывает сумму $N$ независимых и одинаково распределенных в соответствии с $p_{\varepsilon}(x)$ случайных величин $\left\{x_{i}\right\} \quad(i=1, \ldots, N)$. Согласно указанной теореме распределение $p_{\varepsilon}^{(N)}(x)$ в пределе $N \rightarrow \infty$ сходится к распределениям Леви-Хинчина или к нормальному распределению Гаусса в зависимости от значений показателя $\tilde{\delta}_{\varepsilon}(h)$ (или, что то же, значений $\varepsilon$ при данном $h$; подробнее см. раздел 3 приложения).

Для построения РСМ в любом из вариантов - каноническом, Цаллиса или Реньи, обозначаемых соответственно верхним индексом $0, \mathrm{~T}$ или $\mathrm{R},-$ необходимо вычислить функцию Масье-Планка $\Phi$ и энтропию $S$, выражающиеся, как будет показано, через нормировки основного $Z(\beta)$ и сопровождающего $Q(\beta)$ распределений. Ниже в разделе 12 для всех трех вариантов на основе $\Phi(\beta)$ будут получены средняя энергия $E(\beta)$, или калорическое уравнение состояния, а также восприимчивость $\chi(\beta)=\partial E(\beta) / \partial \beta$, или “теплоемкость"

$$
C(T)=\frac{\partial E(T)}{\partial T}, \quad C(T)=-\chi(\beta) \beta^{2}
$$


где учтено, что $\partial / \partial T=-\beta^{2}(\partial / \partial \beta)$.

Кроме того, следует учесть, что в обобщенной $\mathrm{PCM}$, как уже отмечалось в разделе 4 , нарушается присушее канонической РСM [4] свойство пропорциональности теплоемкости флуктуациям энергии, т.е.

$$
-\chi=C(T) T^{2} \neq \sigma_{E}^{2}, \quad \sigma_{E}^{2}=\overline{E^{2}}-\bar{E}^{2} .
$$

Поэтому необходимо наряду с величиной (160) вычислить также независимо величину $\overline{E^{2}} / E^{2}$, согласно (15) определяюшую дисперсию энергии, и с ее помошью установить условия выполнения термодинамической устойчивости (16).

Причина несовпадения величин - $\chi$ и $\sigma^{2}$, отличающего обобщенную РСМ и исчезающего в пределе $\varepsilon=0$, достаточно очевидна и обусловлена свойством (53) обобщенного распределения $p_{\varepsilon}(x)$ из (56) (см. в связи с этим замечание перед формулой (59)). Дело в том, что повторное дифференцирование по $\beta$ (или, в более общем случае, по любой из переменных $\alpha_{k}$, входяших в определяюшую распределение (56) величину $b$ из (30)) выражения для средней энергии

$$
E_{\varepsilon}(\beta)=\int d x E(x) \Pi_{1}\left[\tilde{p}_{\varepsilon}(x)\right]
$$

приводит к выражению

$$
\frac{\partial E_{\varepsilon}(\beta)}{\partial \beta}=-\beta \int d x(\Delta E(x))^{2} \Pi_{2}\left[\tilde{p}_{\varepsilon}(x)\right],
$$

где

$$
\Delta E(x) \equiv E(x)-E_{\varepsilon}(\beta) .
$$

Очевидно, что при переходе от (162) к (163) происходит замена принятого при построении обобшенной $\mathrm{PCM}$ сопровождающего распределения $\Pi_{1}\left(\tilde{p}_{\varepsilon}\right)=\left(\tilde{p}_{\varepsilon}\right)^{\varepsilon+1}$ другим распределением $\Pi_{2}\left(\tilde{p}_{\varepsilon}\right)=\Pi_{1}\left(\tilde{p}_{\varepsilon}\right)(1+\varepsilon)\left(\tilde{p}_{\varepsilon}\right)^{\varepsilon}$, причем в обшем случае $\Pi_{2}\left(\tilde{p}_{\varepsilon}\right) \neq \Pi_{1}\left(\tilde{p}_{\varepsilon}\right)$ (очевидно, что $\Pi_{2}\left(\tilde{p}_{\varepsilon}\right)=\Pi_{1}\left(\tilde{p}_{\varepsilon}\right)=\tilde{p}_{\varepsilon}$ только в пределе $\left.\varepsilon=0\right)$. Подобная замена противоречит естественному требованию универсального определения всех средних величин с одной и той же функцией распределения (см. также формулы (17) и (18)). Поэтому правая часть (163) не может рассматриваться как среднее значение величины $[\Delta E(x)]^{2}$ (дисперсии энергии), которая ниже будет вычисляться в соответствии с обшими формулами (17) и (18),

$$
\sigma_{E}^{2} \equiv \overline{(\Delta E)^{2}}=\int d x(\Delta E(x))^{2} \Pi_{1}\left[\tilde{p}_{\varepsilon}(x)\right],
$$

где согласно определению (133) величины $\Pi_{1}\left(\tilde{p}_{\varepsilon}\right)$ для вариантов Цаллиса и Реньи отличаются множителем $Q_{\varepsilon}$.

Для проведения вычислений с целью построения обобщенной РСМ в рамках принятой модели следует заметить, что все интересующие нас величины являются моментами равновесного распределения (154) (или (155) при $\varepsilon=0$ ) и с учетом ограничения $x \geqslant 0$ представляют собой меллин-образы конечного порядка с ядром (154),

$$
g_{\varepsilon}^{(s)}(h, \nu) \equiv \int_{0}^{\infty} x^{s-1}\left[\tilde{p}_{\varepsilon}(x)\right]^{\nu} d x=g_{0}^{(s)}(\beta, h) \gamma_{\varepsilon}^{(s)}(h, \nu),
$$


где

$$
\begin{aligned}
& g_{0}^{(s)}(\beta, h)=\int_{0}^{\infty} x^{s-1} \tilde{p}_{0}(x) d x=\frac{1}{h} \Gamma\left(\frac{s}{h}\right)\left[x_{0}(\beta)\right]^{s}, \\
& \gamma_{\varepsilon}^{(s)}(h, \nu)=\varepsilon^{-s / h} \Gamma\left(\frac{\nu}{\varepsilon}-\frac{s}{h}\right)\left[\Gamma\left(\frac{\nu}{\varepsilon}\right)\right]^{-1},
\end{aligned}
$$

где $Г(z)$ - гамма-функция, определенная лишш для $z>0$.

Моменты определяются различными наборами параметров $(s, \nu)$ (величины $h$ и $\varepsilon$ предполагаются заданными), причем условия сходимости интегралов (164) определяются условиями существования гамма-множителей в правых частях выражений (165) и (166), что дает соответственно

$$
\frac{s}{h}>0, \quad \frac{\nu}{\varepsilon}>0, \quad \frac{\nu}{\varepsilon}>\frac{s}{h}
$$

Для всех интересующих нас случаев имеем $\nu>0, s \geqslant 1$, так что, учитывая $h>0$, получаем из (167) ограничение сверху на $\varepsilon$ (ограничение снизу $0 \leqslant \varepsilon$ принято нами изначально при выборе знака величины $b(x)$ в $(61))$,

$$
0 \leqslant \varepsilon<\frac{\nu h}{s} \text {. }
$$

Заметим, что в каноническом пределе $\varepsilon=0$ с учетом асимптотических свойств гамма-функции при вещественных $z=x$

$$
\Gamma(x) \approx \sqrt{2 \pi} x^{x-1 / 2} e^{-x}, \quad x \rightarrow \infty,
$$

при любых положительных значениях $s, h, \nu$, удовлетворяюших условиям (168), имеем

$$
\gamma_{\varepsilon}^{(s)}(h, \nu) \rightarrow 1, \quad \varepsilon \rightarrow 0
$$

При вычислении моментов $m_{\varepsilon}^{(n)}$ исходного распределения $p_{\varepsilon}(x)$ используется набор параметров $(s, \nu)=(n+1,1)$. Поэтому согласно общему ограничению $(168)$ условием сушествования этих моментов является неравенство

$$
0 \leqslant \varepsilon<\frac{h}{n+1}
$$

в точности совпадаюшее с условием (П.27), полученным на основе качественного анализа асимптотики $p_{\varepsilon}(x)$. Для дальнейшего сушественной является лишь величина $Z_{\varepsilon} \equiv$ $m_{\varepsilon}^{(0)}$ (нормировка основного распределения), отвечаюшая набору $(1,1)$ при $n=0$. Поэтому мы ограничимся здесь лишь условием

$$
0 \leqslant \varepsilon<h
$$

которое будет далее заменено более ограничительным, следующим из требования конечности дисперсии энергии $\sigma_{E}^{2}$. Заметим, что согласно $(171)$ "кинематический” показатель $\varepsilon$ должен быть всегда строго меньше “динамического" $h$. 
Соотношения (164)-(166) указывают на то, что вся зависимость моментов любого порядка (164) от термодинамического параметра $\beta$ в обобшенной РСМ определяется только множителем (165) с $\varepsilon=0$, тогда как вся "кинематическая" зависимость от $\varepsilon \neq 0$ вносится множителем (166) (“динамический” показатель $h$ входит в оба множителя).

Помимо величины $Z_{\varepsilon}$ в обобщенной $\mathrm{PCM}$ интерес представляет также и величина $Q_{\varepsilon}$ (нормировка сопровождаюшего распределения), вьгисление которой соответствует набору $(1,1+\varepsilon)$, очевидно, менее ограничительному по сравнению с (171).

Аналитические выражения для величин $Z_{\varepsilon}$ и $Q_{\varepsilon}$ имеют вид

$$
\begin{array}{ll}
Z_{\varepsilon}(\beta, h)=Z_{0}(\beta, h) \gamma_{\varepsilon}^{(1)}(h, 1), & Z_{0}(\beta, h)=x_{\mathrm{c}}(\beta) \frac{1}{h} \Gamma\left(\frac{1}{h}\right), \\
Q_{\varepsilon}(\beta, h)=\left[\widetilde{Z}_{\varepsilon}(\beta, h)\right]^{-\varepsilon}, & \widetilde{Z}_{\varepsilon}(\beta, h)=Z_{\varepsilon}(\beta, h) \xi_{\varepsilon}(h),
\end{array}
$$

где

$$
\xi_{\varepsilon}(h)=\left(1-\frac{\varepsilon}{h}\right)^{-1 / \varepsilon}, \quad \xi_{\varepsilon}(h)>1, \quad \xi_{0}(h)=e^{1 / h} .
$$

При получении формул (173) использовано обусловленное свойством гамма-функции $\Gamma(z+1)=z \Gamma(z)$ соотношение

$$
\gamma_{\varepsilon}^{(s)}(h, \nu+\varepsilon)=\left(1-\frac{\varepsilon s}{h \nu}\right) \gamma_{\varepsilon}^{(s)}(h, \nu), \quad \frac{\varepsilon s}{h \nu}<1 .
$$

Для дальнейшего удобно ввести показатель

$$
\delta_{\varepsilon}(h) \equiv\left[\xi_{\varepsilon}(h)\right]^{-\varepsilon}=1-\frac{\varepsilon}{h}, \quad 0<\delta_{\varepsilon} \leqslant 1, \quad \delta_{0}=1,
$$

связанный с ранее введенным в $(158)$ показателем $\tilde{\delta}_{\varepsilon}(h)$ простым соотношением

$$
\delta_{\varepsilon}(h)=\tilde{\delta}_{\varepsilon}(h) \frac{\varepsilon}{h}, \quad \frac{\delta_{\varepsilon}(h)}{\tilde{\delta}_{\varepsilon}(h)}<1 .
$$

\section{2. ОБОБЩЕННАЯ РСМ ДЛЯ ОДНОМЕРНОГО ИДЕАЛЬНОГО ГАЗА. КАНОНИЧЕСКИЙ ВАРИАНТ И ВАРИАНТЫ ЦАЛЛИСА И РЕНЬИ}

Применим полученные в разделе 11 результаты для принятой модели вероятностного объекта типа идеального газа к построению канонического варианта РСМ, а также двух вариантов (Цаллиса и Реньи) обобшенной РСМ. Напомним, что эти варианты строятся на одном и том же обобшенном равновесном распределении (56), но различаются определением энтропийных функционалов (91) и (119) и средних значений (107) и (111). В рамках каждого из этих трех вариантов будут получены равновесная энтропия и обобшенная функция Масье-Планка, а на этой основе - термодинамические уравнения состояния, теплоемкость и дисперсия энергии. Будет также проверено вьполнение соотношений Гиббса-Гельмгольца. 
12.1. Канонический вариант РСМ. Как следует из результатов раздела 4 (уравнения (34) и (35)), при $\varepsilon=0$ для функции Масье-Планка с учетом свойства (36), определения (153) и второго из выражений (172) имеем

$$
\Phi_{0}(\beta, h)=\ln Z_{0}(\beta, h)=-\frac{1}{h} \ln \beta .
$$

Эта функция определена с точностью до слагаемых, не содержащих $\beta$ и в данном случае несушественных. Дифференцируя (178) по $\beta$, находим калорическое уравнение состояния в виде

$$
E_{0}(\beta, h)=-\frac{\partial \Phi_{0}(\beta, h)}{\partial \beta}=\frac{1}{h \beta}, \quad-\bar{b}^{(0)}=\beta E_{0}(\beta, h)=\frac{1}{h},
$$

откуда, в частности, следует представление

$$
\xi_{\varepsilon}(h)=\left(1+\varepsilon \bar{b}^{(0)}\right)^{-1 / \varepsilon}
$$

для функции $\xi_{\varepsilon}(h)$ из (174).

Что касается энтропии $S_{0}$, то ее связывает с $\Phi_{0}$ преобразование Лежандра $(34)$. Выражая с помощью (179) $\beta$ через $E_{0}$ и учитьвая явньй вид $\bar{b}^{0}$, находим (с точностью до несущественных постоянных)

$$
S_{0}\left(E_{0}\right)=\frac{1}{h} \ln E_{0}, \quad \frac{\partial S_{0}\left(E_{0}\right)}{\partial E_{0}}=\frac{1}{h E_{0}}=\beta,
$$

так что очевидным образом выполняется соотношение Клаузиуса (38).

Дифференцируя (178) по $\beta$ повторно, находим термодинамическую восприимчивость

$$
\chi_{0}(\beta, h)=\frac{\partial E_{0}(\beta, h)}{\partial \beta}=-\frac{\partial^{2} \Phi_{0}(\beta, h)}{\partial \beta^{2}}=-\frac{1}{h \beta^{2}},
$$

откуда для теплоемкости получаем

$$
C_{0}(T)=-\chi_{0} \beta^{2}=\frac{1}{h}=\mathrm{const}, \quad E_{0}(T, h)=C_{0} T .
$$

Величина $E_{0}$ представляет собой внутреннюю энергию, приходящуюся на одну (в данном случае единственную) степень свободы объекта в модели идеального газа. В частности, для обычного классического атомно-молекулярного газа следует положить $h=2$, что приводит к известной теореме о равнораспределении $E_{0}=T / 2\left(k_{\mathrm{B}}=1\right)$.

Поскольку при $\varepsilon=0$ теплоемкость пропорциональна флуктуациям энергии, имеем для абсолютной дисперсии энергии

$$
\sigma_{E}^{2}=-\chi_{0}=C_{0} T^{2}>0,
$$

что свидетельствует о термодинамической устойчивости объекта при всех $h$ и $T$. Для дальнейшего полезна и другая форма записи соотношения (184). Именно, для относительной дисперсии имеем с учетом (183)

откуда

$$
\tilde{\sigma}_{E}^{2} \equiv \frac{\sigma_{E}^{2}}{E_{0}^{2}}=\frac{{\overline{E^{2}}}^{(0)}}{E_{0}^{2}}-1=\frac{1}{C_{0}}=h>0,
$$

$$
\frac{\overline{E^{2}}(0)}{E_{0}^{2}}=1+h>1
$$


12.2. Вариант РСМ по Цаллису. Равновесная энтропия в этом случае дается функционалом (91), в который следует подставить равновесное распределение (154). Учитывая выражение $(173)$ для $Q_{\varepsilon}$, результат удобно записать в виде $\left.{ }^{14}\right)$

$$
S_{\varepsilon}=-\frac{1}{\varepsilon}\left[Q_{\varepsilon}-1\right]=\Psi_{-\varepsilon}\left(\widetilde{Z}_{\varepsilon}\right) .
$$

Заметим, что величина $\widetilde{Z}_{\varepsilon}$ в соответствии с определением (120) совпадает с энтропией $S^{\mathrm{HLP}}$.

Применяя далее к $S_{\varepsilon}$ из (187) формулу (100), получаем

$$
S_{\varepsilon}=\Psi_{-\varepsilon}\left(Z_{\varepsilon}\right)+\Psi_{-\varepsilon}\left(\xi_{\varepsilon}\right)\left[1-\varepsilon \Psi_{-\varepsilon}\left(Z_{\varepsilon}\right)\right]=\Psi_{-\varepsilon}\left(Z_{\varepsilon}\right)+\left(Z_{\varepsilon}\right)^{-\varepsilon} \Psi_{-\varepsilon}\left(\xi_{\varepsilon}\right) .
$$

Нетрудно видеть, что выражение (188) допускает предельный переход к канонической равновесной энтропии (34) при $\varepsilon=0$, поскольку с учетом свойства (76) имеем

$$
S_{\varepsilon} \rightarrow S_{0}=\ln \widetilde{Z}_{0}=\ln Z_{0}+\ln \xi_{0}, \quad \xi_{0}=e^{-\bar{b}^{(0)}} .
$$

По аналогии с каноническим вариантом РСМ и в соответствии с общим представлением (148) и (149) покажем, что первое слагаемое в правой части (188) также является функцией Масье-Планка $\Phi_{\varepsilon}(\beta)$, поскольку оно обладает производяшим свойством $(150)$

$$
\Phi_{\varepsilon}=\Psi_{-\varepsilon}\left(Z_{\varepsilon}\right), \quad \frac{\partial \Phi_{\varepsilon}}{\partial \beta}=-E_{\varepsilon}
$$

в чем можно убедиться непосредственно. Действительно, опуская для краткости аргумент $\beta$ у $Z_{\varepsilon}(\beta)$, имеем

$$
\frac{\partial}{\partial \beta}\left[\Psi_{-\varepsilon}\left(Z_{\varepsilon}\right)\right]=\left(Z_{\varepsilon}\right)^{-(\varepsilon+1)} \frac{\partial Z_{\varepsilon}}{\partial \beta}=-E_{\varepsilon}
$$

где использовано определение (107) для частного случая (151) и в соответствии с (46) учтено, что при $n=1$

$$
\frac{\partial Z_{\varepsilon}}{\partial \beta}=-\int E(x)\left[\tilde{p}_{\varepsilon}(b)\right]^{\varepsilon+1} d x=-\left(Z_{\varepsilon}\right)^{\varepsilon+1} \int E(x)\left[p_{\varepsilon}(b)\right]^{\varepsilon+1} d x .
$$

В соответствии с мультипликативной структурой $Z_{\varepsilon}(\beta)$ из $(172)$ получаем

$$
Z_{\varepsilon}^{-1} \frac{\partial Z_{\varepsilon}}{\partial \beta}=\frac{\partial}{\partial \beta} \ln Z_{\varepsilon}(\beta)=\frac{\partial}{\partial \beta} \ln Z_{0}(\beta)=-E_{0}(\beta),
$$

где $E_{0}(\beta)$ дается выражением (179). Окончательно формула для ненормированной обобщенной средней энергии принимает вид

$$
E_{\varepsilon}(\beta)=\left[Z_{\varepsilon}(\beta)\right]^{-\varepsilon} E_{0}(\beta)
$$

\footnotetext{
14) Всюду в этом разделе для простоты записи у всех функций и функционалов будут, как правило, опущены аргументы $x$ и $p(x)$, а также верхний индекс Т, отличающий вариант Цаллиса.
} 
и в пределе $\varepsilon=0$ обрашается в тождество.

Что касается второго слагаемого в правой части (188), то, как и следовало ожидать в соответствии с обшим представлением (149), оно равно $-\bar{b}^{\mathrm{T}}$. Действительно, из формулы (180) следует

$$
\Psi_{-\varepsilon}\left(\xi_{\varepsilon}\right)=-\bar{b}^{(0)}=\beta E_{0}(\beta),
$$

что с учетом выражения (194) приводит к искомому результату

$$
S_{\varepsilon}\left(E_{\varepsilon}\right)-\Phi_{\varepsilon}(\beta)=-\bar{b}^{\mathrm{T}}, \quad \frac{\partial S_{\varepsilon}}{\partial E_{\varepsilon}}=\beta
$$

В явном виде калорическое уравнение состояния (194) можно записать следуюшим образом:

$$
E_{\varepsilon}(\beta, h)=\frac{1}{h} B_{\varepsilon}(h) \beta^{-\delta_{\varepsilon}(h)}, \quad B_{\varepsilon}(h)>0, \quad B_{0}(h)=1 .
$$

Очевидно, в отличие от (183) связь между $E_{\varepsilon}$ и $T=1 / \beta$ становится нелинейной (степенной). Беря логарифмические производные от обеих частей (197), получаем полезное соотношение

$$
E_{\varepsilon} \frac{\partial \beta}{\partial E_{\varepsilon}}=-\frac{1}{\delta_{\varepsilon}} \beta
$$

Напомним, что в каноническом случае $\delta_{0}=1$, тогда как в варианте Цаллиса $0<\delta_{\varepsilon} \leqslant 1$. Дифференцируя (197) по $\beta$, находим

$$
\chi_{\varepsilon}(\beta, h)=\frac{\partial E_{\varepsilon}(\beta, h)}{\partial \beta}=-\frac{\delta_{\varepsilon}(h)}{h} B_{\varepsilon}(h) \beta^{-\left[\delta_{\varepsilon}(h)+1\right]},
$$

откуда для теплоемкости $C_{\varepsilon}(T)$ получаем

$$
C_{\varepsilon}(T, h)=\frac{\delta_{\varepsilon}(h)}{h} B_{\varepsilon}(h) T^{-1+\delta_{\varepsilon}(h)} .
$$

Поскольку $\delta_{\varepsilon}<1$, это означает убывание $C_{\varepsilon}$ с ростом $T$ и, следовательно, качественно отличает случай $\varepsilon \neq 0$ от канонического. Заметим, что теплоемкость $C_{\varepsilon}$ остается при этом положительной при всех $T$, так что термодинамическая устойчивость в обычном смысле не нарушается.

Однако относительную дисперсию энергии $\sigma_{E}^{2}$ необходимо находить независимо, согласно определению (14). Используя общие выражения (164), нетрудно показать, что (в сокрашенной записи)

$$
\frac{{\overline{E^{2}}}^{\mathrm{T}}}{E_{\varepsilon}^{2}}=\frac{{\overline{E^{2}}}^{(0)}}{E_{0}^{2}}\left(Z_{\varepsilon}\right)^{\varepsilon}\left[1-\frac{\varepsilon}{\varepsilon_{\mathrm{\kappa p}}(h)}\right]^{-1}
$$

(при $\varepsilon=0$ выражение (201) переходит в (186)). Тогда условие устойчивости (14) относительно фолуктуаций энергии может быть выполнено только при условии

$$
\varepsilon<\varepsilon_{\mathrm{Kp}}(h), \quad \varepsilon_{\mathrm{Kp}}(h)=\frac{h}{1+h},
$$

которое с учетом неравенства $h>0$ налагает на возможные значения $\varepsilon$ более жесткое ограничение сверху, чем (171). В частности, отсюда следует, что минимальными значениями (точнее, отличными от нуля границами снизу) для введенных в (158) и (177) показателей $\tilde{\delta}_{\varepsilon}(h)$ и $\delta_{\varepsilon}(h)$, являются соответственно $h$ и $1 /(1+h)$. 
12.3. Вариант РСМ по Реньи. Равновесная энтропия в этом случае с учетом исходного определения (117), а также выражений (119) и (180) имеет вид

$$
S_{\varepsilon}^{\mathrm{R}} \equiv \widehat{R}_{\varepsilon}^{-} S_{\varepsilon}^{\mathrm{T}}=-\frac{1}{\varepsilon} \ln Q_{\varepsilon}=\ln \widetilde{Z}_{\varepsilon}=\ln Z_{\varepsilon}+\ln \xi_{\varepsilon} .
$$

При $\varepsilon=0$ предел выражения (203) в точности совпадает с пределом (189) для варианта РСМ по Цаллису (и, следовательно, с каноническим). Заметим, что выражение (203) может быть получено также непосредственно с использованием свойства (116) оператора $\widehat{R}^{-}$, в частности, второе слагаемое правой части (203) имеет вид $\widehat{R}^{-}\left[b^{0}\right]$.

По аналогии как с каноническим вариантом, так и с вариантом Цаллиса первое слагаемое в правой части (203)

$$
\Phi_{\varepsilon}^{\mathrm{R}} \equiv \widehat{R}_{\varepsilon}^{-} \Phi_{\varepsilon}=\ln Z_{\varepsilon}
$$

допустимо рассматривать в качестве функции Масье-Планка в варианте Реньи, если оно обладает производящим свойством

$$
\frac{\partial \Phi_{\varepsilon}^{\mathrm{R}}}{\partial \beta_{\varepsilon}^{\mathrm{R}}}=-E_{\varepsilon}^{\mathrm{R}} .
$$

Это свойство действительно имеет место при выполнении условия

$$
\beta_{\varepsilon}^{\mathrm{R}}=\beta \delta_{\varepsilon}(h), \quad 0 \leqslant \beta_{\varepsilon}^{\mathrm{R}}<\beta,
$$

в необходимости которого нетрудно убедиться следуюшим образом. Объединяя формулы (194) и (173) и учитывая соотношение (133), связываюшее средние значения в двух вариантах обобщенной РСМ, находим для правой части (205) выражение

$$
E_{\varepsilon}^{\mathrm{R}}=Q_{\varepsilon}^{-1} E_{\varepsilon}^{\mathrm{T}}=\widetilde{Z}_{\varepsilon}^{\varepsilon} Z_{\varepsilon}^{-\varepsilon} E_{0}=\left(\xi_{\varepsilon}\right)^{\varepsilon} E_{0}=E_{0}\left[\delta_{\varepsilon}(h)\right]^{-1} .
$$

Учитывая далее формулу (193), окончательно приходим к равенству (205) при условии (206).

Таким образом, обратная температура в варианте РСМ по Реньи отличается от соответствуюшей универсальной величины в двух других вариантах лишь положительным и не превосходяшим единицу масштабным множителем $\delta_{\varepsilon}(h)$. Условие $(206)$ является, по сушеству, условием самосогласования вариантов РСМ по Цаллису и Реньи, поскольку построение Реньи не является вполне последовательным: в нем используется то же распределение (56), что и в варианте Цаллиса. Более подробное обсуждение нецелесообразности использования равновесного распределения, соответствуюшего условному максимуму энтропии Реньи, было приведено в разделе 9.

Несмотря на сушествование функции Масье-Планка (204), соотношение (203) в варианте Реньи, в отличие от двух других вариантов РСМ, не является точным аналогом преобразования Лежандра (149), поскольку второе слагаемое в правой части (203) отлично от $\left(-\bar{b}^{\mathrm{R}}\right)$,

$$
\ln \xi_{\varepsilon}=\widehat{R}^{-}\left[-\bar{b}^{(0)}\right]=-\frac{1}{\varepsilon} \ln \left(1+\varepsilon \bar{b}^{(0)}\right) .
$$


Тем не менее в рамках рассматриваемой модели оказывается, что производяшее свойство энтропии (соотношение Клаузиуса)

$$
\frac{\partial S_{\varepsilon}^{\mathrm{R}}}{\partial E_{\varepsilon}^{\mathrm{R}}}=\beta_{\varepsilon}^{\mathrm{R}}
$$

все же имеет место благодаря следуюшему из формулы (205) простому (квазиканоническому) виду калорического уравнения состояния

$$
E_{\varepsilon}^{\mathrm{R}}=\left(h \beta_{\varepsilon}^{\mathrm{R}}\right)^{-1}, \quad-\bar{b}^{\mathrm{R}}=-\bar{b}^{(0)}=h^{-1},
$$

при котором теплоемкости в вариантах Реньи и каноническом совпадают,

$$
C_{\varepsilon}^{\mathrm{R}}=C_{0}=h^{-1}=\text { const . }
$$

Действительно, пусть энтропия $S(E)$ и функция Масье-Планка $\Phi(\beta)$ какого-либо термодинамически равновесного вероятностного объекта связаны соотношением

$$
S(E)-\Phi(\beta)=\varphi(b),
$$

где $\varphi$ - произвольная дифференцируемая функция (в случае обычного преобразования Лежандра (149) $\varphi$ является однородной линейной функцией, $\varphi(b)=-b)$. Беря дифференциалы обеих частей (212) и учитывая, что в силу (205) $d \Phi=-E d \beta$, находим, что

$$
\frac{d S}{d E}=-E \frac{d \beta}{d E}\left(1+\frac{d \varphi}{d b}\right)-\beta \frac{d \varphi}{d b},
$$

откуда очевидно,что производяшее свойство для равновесной энтропии

$$
\frac{d S}{d E}=\beta
$$

выполняется при любом виде калорического уравнения состояния $E(\beta)$, если $\varphi(b)=-b$, $d \varphi(b) / d b=-1$. Если же $d \varphi(b) / d b \neq-1$, то соотношение (214) выполняется лишь в частном случае, когда калорическое уравнение состояния обладает свойством

$$
E \frac{d \beta}{d E}=-\beta, \quad E(\beta)=C \beta^{-1} .
$$

Именно этот случай, как видно из (210) и (211), реализуется в РСМ Реньи, для которой согласно (203), (208) и второму из равенств (210) имеем

$$
\varphi_{\varepsilon}^{\mathrm{R}}(b)=-\frac{1}{\varepsilon} \ln (1+\varepsilon b) .
$$

Заметим далее, что в случае Реньи по той же причине, что и в случае Цаллиса, остается в силе утверждение о нарушении связи между термодинамическими восприимчивостями (в данном случае теплоемкостью) и корреляционными функциями (в данном случае дисперсией энергии) (см. в связи с этим замечание после формулы (162)). Учитьвая, что нормированные средние по Реньи связаны с ненормированными средними по Цаллису соотношением (203), и используя соотношение (195), получаем

$$
\frac{{\overline{E^{2}}}^{\mathrm{R}}}{\left(E_{\varepsilon}^{\mathrm{R}}\right)^{2}}=\frac{{\overline{E^{2}}}^{\mathrm{T}}}{\left(E_{\varepsilon}^{\mathrm{T}}\right)^{2}} Q_{\varepsilon} .
$$

Нетрудно убедиться, что условие устойчивости (16) при выполнении условия (202) выполняется при любых $h$ и $\varepsilon$ и в отличие от случая Цаллиса не зависит от температуры. 


\section{3. ЗАКЛЮЧЕНИЕ}

В данной работе предпринята попытка дать последовательный анализ следствий, возникающих при $\varepsilon$-деформации "канонического" энтропийного функционала БольцманаГиббса-Шеннона, на основе которого строится каноническая РСМ в рамках принципа максимума информационной энтропии для вероятностных объектов, описываемых непрерывными вероятностными распределениями.

Обобщение канонической РСМ, или построение РСМ “общего положения" (по терминологии Фаддеева [29]), возможно в различных вариантах в зависимости от выбора конкретного энтропийного функционала, а также от способа определения средних значений с помощью сопровождающего распределения, нелинейным степенным образом связанного с основным (исходным) вероятностным распределением.

Результаты работы показывают, что подобная $\varepsilon$-деформация затрагивает многие аспекты канонической РСМ. Поэтому представляется целесообразным привести эти результаты в виде таблицы, снабдив ее некоторыми комментариями. Следует заметить, что общий сравнительный анализ различных вариантов обобщенной РСМ для непрерывных распределений, прежде всего анализ варианта РСМ по Реньи, до настояшего времени не проводился. Ряд результатов для дискретных распределений в варианте РСМ по Цаллису можно найти в обзорных статьях [18] и монографии [19].

\begin{tabular}{|c|c|c|c|c|c|c|c|c|c|c|c|c|c|}
\hline Вариант & \multicolumn{110}{|c|}{ Свойства } \\
\hline & I & II & III & IV & V & VI & VII & VIII & IX & X & XI & XII & XIII \\
\hline Канонический & + & + & + & + & + & + & - & + & + & + & + & + & + \\
\hline Цаллиса & + & + & - & - & - & - & + & + & + & + & - & - & - \\
\hline Реньи & + & + & + & - & + & - & + & $-/+$ & $-/+$ & - & - & + & + \\
\hline
\end{tabular}

Свойства I-III относятся к свойствам обобщенного энтропийного функционала. Они соответствуют требованиям 1-3 энтропийной аксиоматики и подробно обсуждались в разделах 6-8. Особенно важную роль играет свойство III (не)аддитивности энтропии, которое рассматривалось также в работах [17]-[19], [31]. Свойства IV и V касаются определения средних значений, а именно их линейности по основному распределению (IV) и нормированности (V) (разделы 2,7 и 8). Свойства VI и VII касаются свойств исходного, или основного распределения: связи статистической независимости с независимостью динамической (VI) и наличия степенной асимптотики при больших отклонениях случайной переменной от среднего значения (VII) (эти вопросы рассмотрены в разделах 1 и 3 приложения). Свойства VIII-X характеризуют основные величины, описываюшие PCM: производящее свойство для равновесной энтропии (VIII), производящее свойство для функции Масье-Планка (IX) и наличие тесно связанного с этими свойствами преобразования Лежандра $(\mathrm{X})$ между интенсивными и экстенсивными термодинамическими параметрами ${ }^{15)}$ (разделы 4 и 10-12). Сушественно, что даже при наличии свойства IX в обоих обобщенных вариантах не выполняется свойство XI, состоящее в

15) Знак - / в таблице означает отсутствие свойства в общем случае и его наличие в частном случае вероятностного объекта в рамках одночастичной модели ( "идеальный газ"). 
пропорциональности термодинамических восприимчивостей корреляционным функциям (разделы 4 и 11). В частности, имеет место нарушение свойства XII - пропорциональности теплоемкости объекта дисперсии его энергии (раздел 11). Наконец, в обобщенных вариантах РСМ в принщипе может нарушаться свойство XIII - независимость теплоемкости идеального газа от температуры (раздел 12).

В целом, как видно из таблищы, наиболее близок к каноническому как по свойствам функционала энтропии, так и по термодинамическим следствиям обобщенный вариант PCM по Реньи (за исключением свойства VII основного равновесного распределения). Сушественно, что свойством, отличающим от канонического только обобщенный вариант Цаллиса, является свойство III неаддитивности энтропии, которое не имеет места для подавляющего большинства физических объектов (для объектов иной природы этот вопрос нуждается в специальном исследовании в каждом конкретном случае). Заметим лишь, что принципиальную возможность использования неаддитивных величин (в том числе энтропии) в РСМ впервые рассматривал Ландсберг [31]. Цаллис [17] и ряд его последователей [18], [19] полагают, что супераддитивность возможна для объектов с дальнодействушим гравитационным потенциалом взаимодействия. Особенно показателен в этом смысле пример шварцшильдовских черных дыр, для которых энтропия пропорциональна квадрату адлитивной величины - массы (или, что в данном случае то же, энергии покоя). Возможно, для подобных объектов вообше не выполняется условие динамической независимости (П.5) и, следовательно, условие (П.4) для равновесной функции распределения может быть модифицировано (например, в виде (П.6)).

Наиболее интересным и качественно отличающим оба обобшенных варианта от канонического является свойство VII, касающееся основного вероятностного распределения. Дело в том, что экспоненциальная асимптотика (П.23) вполне удовлетворительно описывает многие вероятностные объекты. Например, ею обладает распределение Максвелла по кинетической энергии для молекул идеального газа. Однако многочисленные и весьма надежные данные наблюдений показывают, что для ряда вероятностных объектов (как правило, нефизической природы [6]-[8], [11]-[14]), а также для некоторых физических объектов [5], [33] асимптотика равновесного статистического распределения объекта по микросостояниям при больших $x$ имеет существенно отличньй от (П.23) степенной характер (П.24). Подобньй характер, как уже отмечалось в разделе 1 , присуш вероятностным распределениям Парето (или Мандельброта-Ципфа-Парето) с медленно убывающим длинным "хвостом" $x^{-(1+s)}$ при конечном значении $s$, тогда как (П.23) формально соответствует пределу $s \rightarrow \infty$. Наличие "хвоста" указывает на значительную роль больших флуктуаций, или вклада относительно "редких" событий в формирование стационарного распределения. Как правило, в эмпирических распределениях [7], [8], [11]-[14] имеет место случай $0 \leqslant s \leqslant 3$ при значительном диапазоне положительных значений $h$. Полезные численные оценки величины $s$ на основе вариационного подхода для широкого класса вероятностных объектов были получены в работе [34]. Частный случай ударной фрагментации твердых тел (при значении $h=2 / 3$ ) рассматривался в [35].

Ясно, что получить асимптотику вида (П.24) для канонического распределения (51) возможно лишш при весьма искусственном предположении о логарифмической зависи- 
мости энергии $E(x)=s \ln \left(x / x_{0}\right)$ от динамической переменной $x$. Более адекватным решением проблемы представляется предпринятый в данной работе систематический анализ иного по сравнению с (51) равновесного вероятностного непрерывного распределения (56) и, следовательно, поиск иного по сравнению с (21) вида для функционала информационной энтропии (например, (91)).

Принципиальным в этой связи представляется вопрос о физическом смысле параметра деформации $\varepsilon$ (или, что то же, показателя Реньи $q=\varepsilon+1)$ и возможности его вычисления, а не извлечения из эмпирических данных (например, по формулам (157) и (158)). Ясно, что переход от канонической РСМ к любому из обобщенных вариантов обусловлен нарушением одного или сразу нескольких лежащих в ее основе предположений. К их числу следует, в частности, отнести следующие:

а) однородность и гладкость фазового пространства $X$, характеризующего вероятностный объект ("кинематический фактор");

б) отсутствие неэкранированного дальнодействия (например, гравитационного), что обеспечивает экспоненциальное спадание пространственных коррелящий и аддитивность энергии и энтропии;

в) отсутствие долговременной памяти, т.е. наличие лишь марковской динамики, что обеспечивает экспоненциальное затухание временных корреляций ( “динамический фактор").

Нарушение "кинематического фактора" обычно интерпретируется как наличие у пространства $X$ мультифрактальной структуры, например, в “иерархической” или "решеточной" форме. Первое означает различие вероятностей для различных ансамблей ( "реплик") объекта на различных пространственных масштабах, а второе приводит к разрывному (скачкообразному) характеру стохастической динамики объекта в $X$. Заметим в этой связи, что энтропия Реньи с точностью до множителя совпадает со спектром размерностей мультифрактального объекта (в частности, при $\varepsilon=-1,0,+1$ она дает соответственно хаусдорфову, информационную и корреляционную размерности [22]).

Существенно, что между различными факторами имеется взаимосвязь. Нарушение “кинематического фактора", например, наличие дискретной, или “решеточной”, структуры пространства $X$, препятствует разложению Крамерса-Мойала для основного кинетического уравнения [2] и получению на этой основе обычных уравнений типа Фоккера-Планка. В связи с этим некоторые авторы [36]-[38] предлагают использовать для получения обобшенного (неканонического) стационарного распределения уравнения типа Фоккера-Планка в дробных производных, учитывающие нелокальность и пространственно-временной скейлинг.

\section{СВОЙСТВА ОБОБЩЕННОГО РАСПРЕДЕЛЕНИЯ}

\section{ПРИЛОЖКЕНИЕ}

\section{1. Умножение}

Пусть два вероятностных объекта описываются динамическими величинами $b_{1}$ и $b_{2}$ и являются статистически независимыми. Тогда их совместная нормированная функция распределения $p(b)$ должна выражаться в виде произведения, так что согласно (70)

$$
p(b)=Z^{-1} \tilde{p}(b)=Z_{1}^{-1} Z_{2}^{-1} \tilde{p}\left(b_{1}\right) \tilde{p}\left(b_{2}\right) .
$$


Очевидно, что для этого необходимо и достаточно, чтобы

$$
\tilde{p}(b)=\tilde{p}\left(b_{1}\right) \tilde{p}\left(b_{2}\right)
$$

так как при этом согласно (44) выполняется и соотношение

$$
Z=Z_{1} Z_{2}
$$

Учитывая выражения (51) и (56), нетрудно получить для функций $\tilde{p}_{0}(b)$ и $\tilde{p}_{\varepsilon}(b)$ следуюшие свойства умножения. В каноническом случае $(\varepsilon=0)$

$$
\tilde{p}_{0}\left(b_{1}\right) \tilde{p}_{0}\left(b_{2}\right)=e^{b_{1}} e^{b_{2}}=e^{b_{1}+b_{2}}=\tilde{p}_{0}\left(b_{1}+b_{2}\right),
$$

т.е. динамическая переменная, описывающая составной объект, является аддитивной,

$$
b=b_{1}+b_{2}, \quad \varepsilon=0,
$$

что естественно рассматривать как динамическую независимость этих объектов. В обобщенном случае $(\varepsilon \neq 0)$ аналогичное произведение имеет вид

$\tilde{p}_{\varepsilon}\left(b_{1}\right) \tilde{p}_{\varepsilon}\left(b_{2}\right)=\left[1-\varepsilon b_{1}\right]^{-1 / \varepsilon}\left[1-\varepsilon b_{2}\right]^{-1 / \varepsilon}=\left[1-\varepsilon\left(b_{1}+b_{2}-\varepsilon b_{1} b_{2}\right)\right]^{-1 / \varepsilon}=\tilde{p}_{\varepsilon}\left(b_{1}+b_{2}-\varepsilon b_{1} b_{2}\right)$,

так что соответствуюшая динамическая переменная

$$
b=b_{1}+b_{2}-\varepsilon b_{1} b_{2}, \quad \varepsilon \neq 0,
$$

не является аддитивной. Отсюда видно, что при $\varepsilon \neq 0$ статистическая независимость двух объектов оказывается возможной в отсутствие динамической независимости (она восстанавливается лишь в пределе $\varepsilon=0$ ).

Заметим, что условие мультипликативности (П.2) для одинаково распределенных статистически независимых вероятностных объектов можно рассматривать как функциональное уравнение. Его конкретные реализации (П.4) и (П.6) (совместно с условиями (П.5) и (П.7)) столь же однозначно определяют каноническое (при $\varepsilon=0)$ и обобщенное (при $\varepsilon \neq 0$ ) распределения, как и дифференциальные уравнения (50) и (53) совместно с универсальным (для всех $\varepsilon$ ) граничным условием (45).

\section{2. Дифференцирование и разложение в ряд}

2.1. Производные по $b$. Из определяюшего функцию $\tilde{p}_{0}(b)$ уравнения $(50)$ следует, что для нее при всех $n \geqslant 1$ выполняется условие $(52)$, тогда как для $\tilde{p}_{\varepsilon}(b)$ согласно уравнению (53) соответствуюшая величина явным образом зависит от $n$,

$$
\frac{\partial^{n} \tilde{p}_{\varepsilon}(b)}{\partial b^{n}}=\chi_{n}(\varepsilon)\left[\tilde{p}_{\varepsilon}(b)\right]^{\varepsilon n+1}, \quad n \geqslant 1,
$$

где

$$
\chi_{n}(\varepsilon)=\prod_{k=0}^{n-1}(k \varepsilon+1), \quad \chi_{0}(\varepsilon) \equiv 1
$$


В частности,

$$
\chi_{1}(\varepsilon)=1, \quad \chi_{2}(\varepsilon)=1+\varepsilon, \quad \chi_{3}(\varepsilon)=1+3 \varepsilon+2 \varepsilon^{2} .
$$

Благодаря выполняющемуся при всех $n \geqslant 1$ свойству

$$
\chi_{n}(0) \equiv 1
$$

правая часть (П.8) при $\varepsilon=0$ переходит в правую часть (52), что свидетельствует о непрерывности по $\varepsilon$ при $\varepsilon \rightarrow 0$ не только самой функции $\tilde{p}_{\varepsilon}(b)$ из $(56)$, но и ее производных по $b$ любого порядка.

В общем случае $\varepsilon \neq 0$ функция (П.9) представляет собой неоднородный многочлен степени $n-1$ по $\varepsilon$,

$$
\begin{gathered}
\chi_{n}(\varepsilon)=\sum_{k=0}^{n-1} \lambda_{k}^{(n)} \varepsilon^{k}, \quad \lambda_{0}^{(n)} \equiv 1, \\
\lambda_{1}^{(n)}=\sum_{k=1}^{n-1} k=\frac{1}{2} n(n-1), \quad \lambda_{n-1}^{(n)}=\prod_{k=1}^{n-1} k=(n-1) ! .
\end{gathered}
$$

Для остальных значений $2 \leqslant k_{\varepsilon} \leqslant n-2$ коэффициенты $\lambda_{k}^{(n)}$ могут быть выражены через биномиальные коэффициенты $C_{k}^{n}$.

Располагая выражением (П.12) для $\chi_{n}(\varepsilon)$ и учитьвая свойство (45), можно представить функцию $p_{\varepsilon}(b)$ в виде ряда Тейлора по степеням $b$,

$$
\tilde{p}_{\varepsilon}(b)=1+\sum_{n=1}^{\infty} \frac{1}{n !} \chi_{n}(\varepsilon) b^{n} .
$$

Полагая $\varepsilon=0$ в правой части (П.13) и учитывая свойство (П.11), мы получаем разложение для $\tilde{p}_{0}(b)=e^{b}$, что свидетельствует о непрерьвности по $\varepsilon$ ряда (П.13).

2.2. Производные по $x$. В ряде случаев, например при определении точек экстремума обобщенной функции распределения $\tilde{p}_{\varepsilon}[b(x)]$, представляет интерес ее производная по исходной случайной переменной $x$. С учетом $(56)$ и $(29)$ имеем

$$
\frac{\partial \tilde{p}_{\varepsilon}[b(x)]}{\partial x}=\frac{\partial \tilde{p}_{\varepsilon}[b(x)]}{\partial[b(x)]} \frac{\partial b(x)}{\partial x}=-\left\{\tilde{p}_{\varepsilon}[b(x)]\right\}^{\varepsilon+1} \sum_{i \geqslant 1} \alpha_{i} \frac{\partial A_{i}(x)}{\partial x},
$$

откуда для определения точек экстремума получаем уравнения

$$
\sum_{i \geqslant 1} \alpha_{i} \frac{\partial A_{i}(x)}{\partial x}=0, \quad \frac{\partial E(x)}{\partial x}=0 .
$$

Второе уравнение в (П.15) соответствует простейшему случаю (151) при $\beta \neq 0$. Обычно функция $E(x)$ при $x \geqslant 0$ является степенной функцией вида (152) (или какой-либо иной монотонно возрастающей функцией), так что уравнение экстремума имеет всего один равный нулю конечный корень.

В более обшем случае, например когда функция $E(x)=A x^{2}+B x^{4}(A<0, B>0)$ описывает двухъямный потенциал, второе уравнение в (П.15) имеет три конечных решения (два минимума и один максимум между ними). Согласно соотношению (П.14) эти точки будут являться экстремальными также и для $p_{\varepsilon}[b(x)]$ при любом $\varepsilon$. 
2.3. Производные по $\varepsilon$. Дифференшируя почленно сходяшийся ряд (П.13) по параметру $\varepsilon$, находим

$$
\frac{\partial^{m} \tilde{p}_{\varepsilon}(b)}{\partial \varepsilon^{m}}=\sum_{n=1}^{\infty} \frac{1}{n !} \frac{\partial^{m} \chi_{n}(\varepsilon)}{\partial \varepsilon^{m}} b^{n}=\sum_{n=m+1}^{\infty} \frac{b^{n}}{n !} \sum_{k=m}^{n-1} \prod_{l=0}^{m-1}(k-l) \lambda_{k}^{(n)} \varepsilon^{k-m},
$$

где мы использовали свойство

$$
\frac{\partial^{m} \chi_{n}(\varepsilon)}{\partial \varepsilon^{m}}=0 \quad \text { при } \quad m \geqslant n .
$$

Вычислить правую часть (П.16) в обшем виде при $\varepsilon \neq 0$ затруднительно, однако при $\varepsilon=0$ она сушественно упрошается, поскольку в частичной сумме по $k$ остается отличным от нуля лишь член низшего порядка с $k=m$. Произведение по $l$ дает при этом $m !$, так что окончательно имеем

$$
\left.\frac{\partial^{m} \tilde{p}_{\varepsilon}(b)}{\partial \varepsilon^{m}}\right|_{\varepsilon=0}=m ! \sum_{n=m+1}^{\infty} \frac{b^{n}}{m !} \lambda_{m}^{(n)} .
$$

Выражение (П.17) остается достаточно сложным. Мы вычислим его в явном виде лишь для $m=1$, когда согласно (П.12) $\lambda_{1}(n)=n(n-1) / 2$. Имеем тогда

$$
\left.\frac{\partial \tilde{p}_{\varepsilon}(b)}{\partial \varepsilon}\right|_{\varepsilon=0}=\frac{1}{2} \sum_{n=2}^{\infty} \frac{b^{n}}{(n-2) !}=\frac{1}{2} \sum_{n=0}^{\infty} \frac{b^{n+2}}{n !}=\frac{1}{2} b^{2} e^{b}=\frac{1}{2}\left[\ln \tilde{p}_{0}(b)\right]^{2} \tilde{p}_{0}(b)>0 .
$$

Ввиду того что возможны ситуации, когда $b=b(\varepsilon)$ (например, при получении совместного распределения (П.6)), целесообразно получить результат более простым способом, а затем применить его к случаю $b=b(\varepsilon)$. С этой целью вычислим логарифмическую производную не ряда (П.13), а его суммы (56). Это дает

$$
\left.\left[\frac{\partial}{\partial \varepsilon} \ln \tilde{p}_{\varepsilon}(b)\right]\right|_{\varepsilon=0}=\left.\frac{\partial}{\partial \varepsilon}\left\{-\frac{1}{\varepsilon} \ln [1-\varepsilon b]\right\}\right|_{\varepsilon=0}=-\left.\frac{1}{2}\left\{\frac{\partial^{2}}{\partial \varepsilon^{2}} \ln [1-\varepsilon b]\right\}\right|_{\varepsilon=0}=\frac{1}{2} b^{2},
$$

откуда, учитывая, что $(\partial / \partial \varepsilon) \ln p_{\varepsilon}=p_{\varepsilon}^{-1}\left(\partial p_{\varepsilon} / \partial \varepsilon\right)$ и $\tilde{p}_{0}(b)=e^{b}$, вновь приходим к (П.18).

Заметим, что если $b=b(\varepsilon)$ в левой части равенства (П.19), то его правая часть должна быть дополнена слагаемым $\partial b(\varepsilon) / \partial \varepsilon$ при $\varepsilon=0$. Для интересующего нас случая (П.7) $b$ зависит от $\varepsilon$ линейно, так что

$$
b(0)=b_{1}+b_{2}, \quad b^{\prime}(0)=-b_{1} b_{2}, \quad \frac{1}{2}\left[b^{2}(0)+2 b^{\prime}(0)\right]=\frac{1}{2}\left(b_{1}^{2}+b_{2}^{2}\right)>0 .
$$

Таким образом, величина (П.18) положительно определена при всех $b$ и, следовательно, знак разности $\Delta p_{\varepsilon}(b)$ в низшем порядке по $\varepsilon$ при любом $b$ определяется только знаком самого параметра $\varepsilon$. 


\section{3. Асимптотика и сходимость моментов}

Рассмотрим поведение $\tilde{p}_{\varepsilon}(b)$ при малых и больших значениях $|b|$ в случаях, когда $b>$ 0 или $b<0$.

3.1. Малые $|b|$ (при любом знаке $b)$. Этот случай фактически уже рассмотрен в п. 2.1, где для $\tilde{p}_{\varepsilon}(b)$ получено тейлоровское разложение (П.13), в котором можно удержать любое требуемое для желаемой точности число членов. Заметим, что поскольку $\chi_{0}(\varepsilon)=\chi_{1}(\varepsilon)=1$, разность между обобшенным и каноническим распределениями

$$
\Delta \tilde{p}_{\varepsilon}(b) \equiv \tilde{p}_{\varepsilon}(b)-\tilde{p}_{0}(b)=\sum_{n=0}^{\infty} \frac{1}{n !}\left[\chi_{n}(\varepsilon)-1\right] b^{n}
$$

при малых $|b|$ становится отличной от нуля, лишь начиная с членов второго порядка по $b$. С учетом того что $\chi_{2}(\varepsilon)=1+\varepsilon$, разность (П.20) имеет приближенное значение

$$
\Delta \tilde{p}_{\varepsilon}(b) \approx \frac{1}{2} \varepsilon b^{2},
$$

тогда как в нулевом и первом порядке по $b$ рассматриваемые распределения совпадают,

$$
\tilde{p}_{\varepsilon}(b) \approx \tilde{p}_{0}(b) \approx 1+b .
$$

3.2. Большие $|b|$. В отличие от случая малых $|b|$ этот случай сушественно зависит от знака $b$, причем поведение обобшенного и канонического распределений различается качественно, что представляет интерес для приложений всего подхода в целом.

В случае $b<0$ при больших значениях $|b|$ каноническое распределение имеет убывающее экспоненциальное поведение,

$$
\tilde{p}_{0}(b) \sim e^{-|b|}, \quad|b| \gg 1
$$

а обобщенное распределение - убывающее степенное поведение,

$$
\tilde{p}_{\varepsilon}(b) \sim(\varepsilon|b|)^{-1 / \varepsilon}, \quad \varepsilon|b| \gg 1
$$

Ясно, что для справедливости (П.24) должно быть выполнено условие $\varepsilon>0$, причем в соотношении (П.24) переход к пределу $\varepsilon=0$ уже невозможен.

3.3. Сходимость моментов. В частном случае (151) для одномерного однокомпонентного распределения (154), заданного на полуоси [0, $)$, асимптотика (П. 24) принимает вид (157) с показателем (158). Эта асимптотика дает возможность качественно определить условия сушествования ненормированных моментов $(n=0,1, \ldots)$

$$
m_{n} \equiv \overline{x^{n}}=\int_{0}^{\infty} d x x^{n} \tilde{p}_{\varepsilon}(x) .
$$

Отсутствие расходимости этих величин на верхнем пределе с учетом асимптотики (157) определяется конечностью предела

$$
\lim _{x \rightarrow \infty} \int d x x^{n} x^{-\left(1+\tilde{\delta}_{\varepsilon}\right)} \sim \lim _{x \rightarrow \infty} x^{n-\tilde{\delta}_{\varepsilon}}
$$


что, в свою очередь, возможно при выполнении условия

$$
n-\tilde{\delta}_{\varepsilon} \leqslant 0, \quad \tilde{\delta}_{\varepsilon} \geqslant n, \quad \varepsilon \leqslant \frac{h}{n+1},
$$

где учтено определение (176). В том случае, если предел (П.26) обращается в нуль, основной вклад в моменты (П.25) определяется значениями $p_{\varepsilon}(x)$ при конечных значенияx $x$.

Заметим, что тот же результат может быть получен и с помощью характеристической функции $g_{\varepsilon}(k)$ (фурье-образа распределения $\left.\tilde{p}_{\varepsilon}(x)\right)$, поскольку

$$
m_{n}=\left.i^{n} \frac{\partial^{n} g_{\varepsilon}(k)}{\partial k^{n}}\right|_{k=0}
$$

а асимптотике $(157)$ для $p_{\varepsilon}(x)$ при $x \rightarrow \infty$ соответствует асимптотика для $g_{\varepsilon}(k)$ при $k \rightarrow 0$ вида

$$
g_{\varepsilon}(k) \approx 1-|k|^{s_{\varepsilon}-1}=1-|k|^{\tilde{\delta}_{\varepsilon}}
$$

так что

$$
\lim _{k \rightarrow 0} \frac{\partial^{n} g_{\varepsilon}(k)}{\partial k^{n}} \sim \lim _{k \rightarrow 0}|k|^{\tilde{\delta}_{\varepsilon}-n}
$$

Ясно, что условие (П.27) конечности предела (П.26) является одновременно и условием конечности предела (П.30). Существенно, что эти “асимптотические” условия сходимости совпадают с точными условиями (168) при $\nu=1, s=n+1$.

Для выяснения условий применимости обобщенной центральной предельной теоремы достаточно убедиться в наличии или отсутствии сходимости низших из моментов (П.25) с $n=0,1$ и 2 . Эти моменты определяют соответственно нормировку $m_{\varepsilon}^{(0)}$, среднее значение $m_{\varepsilon}^{(1)}$ и дисперсию $m_{\varepsilon}^{(2)}$ распределения $p_{\varepsilon}(x)$. Согласно (П.27) условия их сушествования могут быть записаны в виде неравенств, с ростом $n$ все сильнее ограничивающих сверху значения $\varepsilon$,

$$
\varepsilon<h \text { для } m_{0}, \quad \varepsilon<\frac{h}{2} \text { для } m_{1}, \quad \varepsilon<\frac{h}{3} \quad \text { для } m_{2} .
$$

Первое из условий (П.31) (совпадающее с (171)) предполагается выполненным всегда, тогда как второе и (или) третье из них могут нарушаться. Соответственно в области значений $h / 3<\varepsilon<h$ распределение $p_{\varepsilon}^{(N)}(x)$, определенное соотношением $(159)$, при $N \rightarrow \infty$ сходится к одному из распределений Леви-Хинчина с бесконечным средним значением и (или) дисперсией, тогда как при выполнении условия $\varepsilon \leqslant h / 3-$ к нормальному распределению Гаусса с конечными значениями обеих указанных величин.

Соответственно показатель Реньи $q=\varepsilon+1$ для указанных двух классов предельных распределений должен удовлетворять неравенствам $h / 3+1<q<h+1$ или $q \leqslant h / 3+1$, что для наиболее распространенного случая $h=2$ дает $5 / 3<q<3$ или $q \leqslant 5 / 3$. 


\section{4. Интегральное представление}

В ряде случаев может быть полезно интегральное представление обобщенного распределения $p_{\varepsilon}(b)$ из $(56)$ через однопараметрическое семейство нормальных распределений $p_{0}(\lambda b)$, где $\lambda$ - любое вешественное число. Подобное семейство соответствует различным (возможно, случайным) значениям входящих в $b$ интенсивных термодинамических параметров. В однокомпонентном случае (151) соответствуюшее выражение имеет вид

где функция

$$
\tilde{p}_{\varepsilon}(x)=\int_{0}^{\infty} d \lambda f_{\varepsilon}(\lambda ; \beta) e^{-\lambda E(x)},
$$

$$
f_{\varepsilon}(\lambda ; \beta)=\frac{\left[\gamma_{\varepsilon}(\beta)\right]^{\alpha_{\varepsilon}}}{\Gamma(1 / \varepsilon)} \lambda^{\alpha_{\varepsilon}-1} e^{-\gamma_{\varepsilon}(\beta) \lambda}
$$

с параметрами $\gamma_{\varepsilon}(\beta)=(\varepsilon \beta)^{-1}, \alpha_{\varepsilon}=1 / \varepsilon$ является плотностью вероятности гамма-распределения [28].

Выражение (П.32) нетрудно получить, используя известное интегральное представление гамма-функции

$$
\Gamma(\alpha)=\int_{0}^{\infty} t^{\alpha-1} e^{-t} d t .
$$

Произведя в нем замену переменной $t \rightarrow s t, s>0$, находим

$$
s^{-\alpha}=\frac{1}{\Gamma(\alpha)} \int_{0}^{\infty} t^{\alpha-1} e^{-s t} d t .
$$

Полагая далее $s=1+\varepsilon \beta E(x)$ (положительность этой величины гарантирована общим условием (60)), $\alpha \equiv \alpha_{\varepsilon}=1 / \varepsilon$ и вводя переменную $\lambda=t\left[\gamma_{\varepsilon}(\beta)\right]^{-1}$, приходим к формуле (П.32).

Заметим, что если рассматривать переменную $\lambda$ как случайную, то ее среднее значение $\bar{\lambda}$ с функцией распределения (П.33) равно $\alpha_{\varepsilon} / \gamma_{\varepsilon}(\beta)=\beta$, абсолютная дисперсия $\overline{(\Delta \lambda)^{2}} \equiv \overline{(\lambda-\bar{\lambda})^{2}}$ равна $\alpha_{\varepsilon} /\left[\gamma_{\varepsilon}(\beta)\right]^{2}=\varepsilon \beta^{2}$, а относительная дисперсия $\left(\overline{\lambda^{2}} / \bar{\lambda}^{2}-1\right)$ равна $\left(\alpha_{\varepsilon}\right)^{-1}=\varepsilon$. Тогда можно формально рассматривать величину $\varepsilon$ (и, следовательно, показатель Реньи $q=\varepsilon+1)$ как обусловленную флуктуациями температуры объекта, которая лишь в среднем совпадает с температурой термостата $\beta$.

Подобный подход (который рассматривается, например, в [10]) представляется вполне правомерным, поскольку, используя вблизи $\lambda=\bar{\lambda}$ разложение

$$
e^{-\lambda E(x)} \approx e^{-\bar{\lambda} E(x)}\left\{1+(\Delta \lambda) E(x)+\frac{1}{2}(\Delta \lambda)^{2}(E(x))^{2}+\cdots\right\},
$$

получим для (П.32) приближенное выражение

$$
\tilde{p}_{\varepsilon}(x) \approx \tilde{p}_{0}(x)\left\{1+\frac{1}{2} \overline{(\Delta \lambda)^{2}}(E(x))^{2}+\cdots\right\} \approx \tilde{p}_{0}(x)\left\{1+\frac{1}{2} \varepsilon \beta^{2}(E(x))^{2}+\cdots\right\},
$$

совпадаюшее в низшем порядке с разложением по степеням $\varepsilon$ или по степеням $b$, полученным выше в (П.18) и (П.21), соответственно.

Благодарности. Автор выражает искреннюю благодарность А. Д. Суханову заполезные обсуждения, а также Е. Ю. Каринцевой за помощь при подготовке рукописи к печати. 


\section{Список литературы}

[1] K. Гардинер. Стохастические методы в естественных науках. М.: Мир, 1986.

[2] Н. Г. Ван Кампен. Стохастические процессы в физике и химии. М.: Высшая школа, 1990.

[3] E. T. Jaynes. Phys. Rev. 1957. V. 106. P. 620; V. 108. P. 171.

[4] Д. Н. Зубарев. Неравновесная статистическая термодинамика. М.: Наука, 1971.

[5] В. М. Золотарев. Устойчивые законы и их применение. М.: Знание, 1984.

[6] М. Шредер. Фракталы, хаос, степенные законы. Москва-Ижевск: Изд-во РХД, 2001.

[7] Б. А. Трубников. Природа. 1993. № 11. С. 3 ; 1995. № 11. С. 48.

[8] А.П. Левич. Структура экологических сообществ. М.: Изд-во МГУ, 1980; Биофизика. 1997. T. 42. C. 534.

[9] W. M. Alberico, A. Lavagno, P. Quarati. Eur. Phys. J. C. 2000. V. 12. P. 499.

[10] G. Wilk, Z. Wlodarczyk. Phys. Rev. Lett. 2000. V. 84. P. 2770.

[11] Математические методы в социальных науках. Ред. А. И. Яблонский. М.: Мысль, 1973.

[12] K. Черри. Человек и информация. М.: Связь, 1972.

[13] А. И. Яблонский. Математические методы в исследовании науки. М.: Наука, 1986.

[14] Р. Л. Стратонович. Теория информации. М.: Сов. радио, 1975.

[15] E. W. Montroll, M. F. Shlesinger. J. Stat. Phys. 1983. V. 32. P. 209.

[16] A. Renyi. Probability Theory. Amsterdam: North-Holland, 1970.

[17] C. Tsallis. J. Stat. Phys. 1988. V. 52. P. 479.

[18] Nonextensive Statistical Mechanics and Thermodynamics (special issue). Ed. C. Tsallis. Brazil J. Phys. 1999. V. 29. № 1.

[19] S. Abe, Y. Okamoto (Eds.). Nonextensive Statistical Mechanics and Its Applications. Lect. Notes Phys. V. 560. Berlin: Springer, 2001.

[20] J. Aczel, Z. Daroczy. On Measures of Information and Their Characterization. N.Y.: Academic Press, 1975.

[21] G. Wehrl. Rev. Mod. Phys. 1978. V. 50. P. 221.

[22] C. Beck, F. Schlögl. Thermodynamic of Chaotic Systems. Cambridge: Cambridge Univ. Press, 1992.

[23] C. Shannon. Bell Syst. Tech. 1948. V. 27. P. 379, 623.

[24] А.Я. Хинчин. УМН. 1953. Т. 8. С. 3.

[25] Д. К. Фаддеев. УМН. 1956. Т. 11. С. 227.

[26] И. М. Гельфанд, А. Н. Колмогоров, А. М. Яглом. ДАН СССР. 1956. Т. 111. С. 745.

[27] Е. С. Вентчель. Теория вероятностей. М.: Высшая школа, 1998.

[28] Вероятность и математическая статистика. Энциклопедия под ред. Ю. В. Прохорова. М.: Изд-во БРЭ, 1999.

[29] Л. Д. Фаддеев. Природа. 1989. № 5. С. 11.

[30] G. Parisi. J. Phys. 1980. V. 13. P. 1701.

[31] P. T. Landsberg. J. Stat. Phys. 1984. V. 35. P. 159.

[32] Г. Харди, Д. Литлвуд, Дж. Пойа. Неравенства. М.: ИЛ, 1948.

[33] Фракталы в физике. Труды VI международного симпозиума по фракталам в физике (МЦТФ, Триест, Италия, 9-12 июля, 1985). Ред. Я.Г. Синай, И. М. Халатников. М.: Мир, 1988.

[34] A. G. Bashkirov, A. V. Vityazev. Physica A. 2000. V. 277. P. 136.

[35] A. G. Bashkirov, A. V. Vityazev. Planet Space Sci. 1996. V. 44. P. 909.

[36] Р. Р. Нигматуллин. ТМФ. 1992. Т. 90. С. 354.

[37] G. M. Zaslavsky. Physica D. 1994. V. 76. P. 110.

[38] А.Д. Суханов, С. Ф. Тимашев. ЖХХХ. 1998. Т. 72. С. 2088.

Поступила в редакцию 21.XI.2001 г., после доработки 5.VII.2002 г. 